\title{
Magneto-Optical and Magnetic Studies of Co-Rich Glass-Covered Microwires
}

\author{
Alexander Chizhik and Valentina Zhukova \\ Departamento de Fusica de Materiales, Facultad de Quımica, Universidad del Pais Vasco, UPV/EHU, 1072, \\ 20080 San Sebastian, Spain \\ Correspondence should be addressed to Alexander Chizhik, oleksandr.chyzhyk@ehu.es
}

Received 22 July 2011; Revised 10 January 2012; Accepted 18 January 2012

Academic Editor: Mitsuteru Inoue

Copyright ( 92012 A. Chizhik and V. Zhukova. This is an open access article distributed under the Creative Commons Attribution License, which permits unrestricted use, distribution, and reproduction in any medium, provided the original work is properly cited.

The magnetization reversal process in the surface and volume areas of Co-rich glass-covered microwires has been investigated. The study has been performed in the wide series of microwires with chemical composition, geometry (thickness of glass coating) with the purpose of the tailoring of the giant magnetoimpedance effect. The comparative analysis of the magnetoelectric, magnetic, and magneto-optical experiments permits to optimise the giant magnetoimpedance ratio and elucidate the main properties of the magnetization reversal process in the different parts of the Co-rich microwire.

\section{Introduction}

The investigation of the magnetization reversal process in amorphous wires and microwires is one of the most important tasks related to the use of these magnetic wires in different technological devices. In particular, the intensive studies of magnetic properties of nearly zero magnetostriction Corich wires and glass-covered microwires have been performed in relation with the giant magnetoimpedance (GMI) effect [1]. This GMI effect is of great interest in sensor application. As is well known, the origin of the GMI effect is related with the penetration depth of the skin effect. Consequently, the investigation and comparison on the magnetization reversal in the surface and volume areas of the wire become a particular importance.

The present paper is devoted to the recent results on magneto-optical Kerreffect and fluxmetric investigations of the magnetization reversal and domain structure in glasscoated microwires. Having in our laboratory the wide line of the magnetic, magnetoelectric, and magneto-optic experimental techniques, we have created the new direction of the complex study of glass covered microwires. During last years, we have performed series of the magneto-optical investigations of different types of the glass covered microwires. Kerr effect and fluxmetric methods as two complementary methods provide the complex information relatively on the magnetization reversal and magnetic domain structure in the volume and in the surface of the microwires. Analyzing the experimental results obtained by these two methods we could model and predict the time and space distribution and transformation of the tree-dimensional magnetic structure of the cylindrical-shaped microwires.

In turn, the magneto-optical set-up which we use to study the microwires consists of two complementary parts: Kerr effect magnetometer and Kerr effect microscopy. Taking into account non-plane surface of the studied micorwires the application of the Kerr effect technique is accompanied by the specific difficulties which were successfully overcame during the experiments performed in our laboratory. First, the doubt relatively possible application of Kerr effect was related to the possible interference which could be produced by the glass covering. This doubt was resolved: because of extremely thin thickness the glass covering does not present noticeable contribution. Second, the difficult related to the light reflection from the cylindrical surface of the microwire was resolved by the application of the system of lenses and diaphragms. In the same time, application of the Kerr effect microscopy to the study of the non-plane surface of the microwire gave unexpected result: the series of the original 
images of surface domain structure has been obtained. Applying the polar configuration of the Kerr microscopy which is sensitive to magnetization perpendicular to the studied surface, we have obtained unusual, very informative images of bamboo-type domains.

Following originally to the technological task of the optimization and miniaturization of the active elements of the magnetic sensors, we nevertheless focused also our attention on such fundamental problems as magnetic domain formation and transformation and domain walls motion. This duality also reflected in the present paper.

\section{Correlation between the GMI Effect and Bulk and Surface Hysteresis Loops}

Magnetically soft Co-rich glass-coated microwires have been fabricated by the Taylor-Ulitovsky method. Among the Corich compositions a $\mathrm{Co}_{69-x} \mathrm{Mn}_{6+x} \mathrm{Si}_{10} \mathrm{~B}_{15}$ series varying the chemical composition $(x=0 ; 0.5 ; 0,75$, and 1$)$ of the metallic nucleus and $\mathrm{Co}_{67} \mathrm{Fe}_{3.85} \mathrm{Ni}_{1.45} \mathrm{~B}_{11.5} \mathrm{Si}_{14.5} \mathrm{Mo}_{1.7}$ microwires of different geometric ratio, $\rho$, of metallic core diameter to total microwire diameter $0.789 \leq \rho \leq 0.98$ have been fabricated $[2,3]$. The chemical composition has been selected taking into account the studies on the effect of chemical composition on magnetic softness of amorphous alloys $[4,5]$.

The sample composition has been selected among the series of $\mathrm{Co}_{69-x} \mathrm{Mn}_{6+x} \mathrm{Si}_{10} \mathrm{~B}_{15}(0<x<1)$ compositions in order to achieve the best combination of soft magnetic properties (high magnetic permeability, low magnetic anisotropy field, and low coercivity). An increasing of the magnetic permeability and coercivity and decreasing of the magnetic anisotropy field were observed in glass-coated amorphous $\mathrm{Co}_{69-x} \mathrm{Mn}_{6+x} \mathrm{Si}_{10} \mathrm{~B}_{15}(0<x<1)$ compositions with $x$ at $x<0.75$ [6]. The magnetostriction constant of $\mathrm{Co}_{69-x} \mathrm{Mn}_{6+x} \mathrm{Si}_{10} \mathrm{~B}_{15}$ samples changes its sign at around $x=0.75$ [7], being of negative character at $x<0.75$. Small negative magnetostriction constant can be assumed for the sample with $6.5 \%$ at $\mathrm{Mn}$. Accordingly, it is expected that the outer domain structure changes its circular easy magnetization axis (very favourable for the GMI effect) to the radial one at $x \approx 0.75$.

Conventional and surface hysteresis loops have been measured by fluxmetric and Kerr effect (MOKE) [8] methods, respectively. An axial DC-field with intensity up to 200 Oe was supplied by a magnetization coils.

The electrical impedance of the microwire was evaluated by means of the four-point technique. The magnetoimpedance ratio, $\Delta Z / Z$, has been defined as:

$$
\frac{\Delta Z}{Z}=\frac{\left[Z(H)-Z\left(H_{\max }\right)\right]}{Z\left(H_{\max }\right)} .
$$

Bulk hysteresis loops of three magnetically soft glasscoated $\mathrm{Co}_{67} \mathrm{Fe}_{3.85} \mathrm{Ni}_{1.45} \mathrm{~B}_{11.5} \mathrm{Si}_{14.5} \mathrm{Mo}_{1.7}$ microwires with different geometric ratio $0.78 \leq \rho \leq 0.98$ are shown in Figure 1. As can be observed from this figure, the magnetic anisotropy field, $H_{k}$, increases with decreasing ratio $\rho$, that is, with the increase of the glass coating thickness.

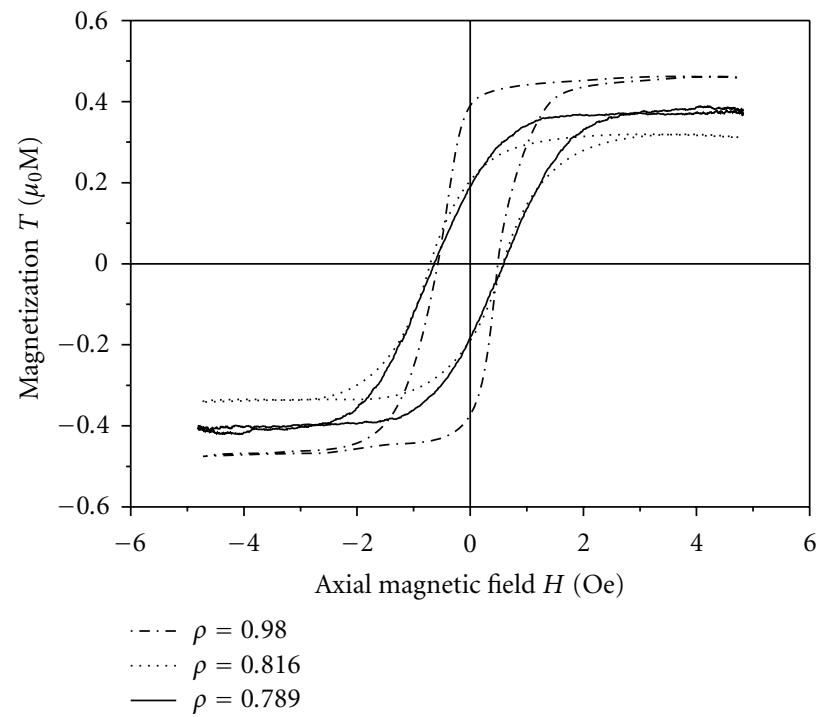

FIgure 1: Bulk hysteresis loop of three samples with $\rho$ as a parameter.

Strong dependence of the hysteresis loops on the parameter $\rho$ should be attributed to the magnetoelastic energy. The values of the internal stresses in glass coated microwires arises from the difference in the thermal expansion coefficients of metallic nucleus and glass coating, depending strongly on the ratio between the glass coating thickness and metallic core diameter, increasing with the increasing of the glass coating thickness. Large internal stresses give rise to a drastic change of the magnetoelastic energy, even for small changes of the glass-coating thickness at fixed metallic core diameter. Additionally, such a change of the $\rho$ ratio should be related to the change of the magnetostriction constant with applied stress.

The dependence of the magnetoimpedance ratio on the axial field at the driving AC-current ranging from 0.75 up to $5 \mathrm{~mA}$ of the frequency, both treated as the parameters have been investigated. The electrical impedance of the microwire was evaluated by means of the four-point technique.

The $(\Delta Z / Z)(H)$ dependences measured at $f=10 \mathrm{MHz}$ and $I=0.75 \mathrm{~mA}$ for the samples with ratio $\rho=0.98,0.816$, and 0.789 are presented in the Figure 2 .

A maximum relative change in the GMI ratio, $\Delta Z / Z$, up to around $615 \%$ is observed at $f=10 \mathrm{MHz}$ and $I=0.75 \mathrm{~mA}$ in the sample with $\rho \approx 0.98$ (see Figure 2).

As may be seen from Figure 2, the field corresponding to the maximum of the GMI ratio, $H_{m}$ increases, and $(\Delta Z / Z)_{m}$ decreases with $\rho$. Such $H_{m}(\rho)$ dependence should be attributed to the effect of internal stresses, $\sigma$, on the magnetic anisotropy field. Indeed, the value of the $D C$ axial field corresponding to the maximum of the GMI ratio, $H_{m}$, should be attributed to the static circular anisotropy field, $H_{k}[1,9]$. The estimated values of the internal stresses in these amorphous microwires are of the order of $1000 \mathrm{MPa}$, depending strongly on the thickness of glass coating and metallic nucleus radius [10]. 


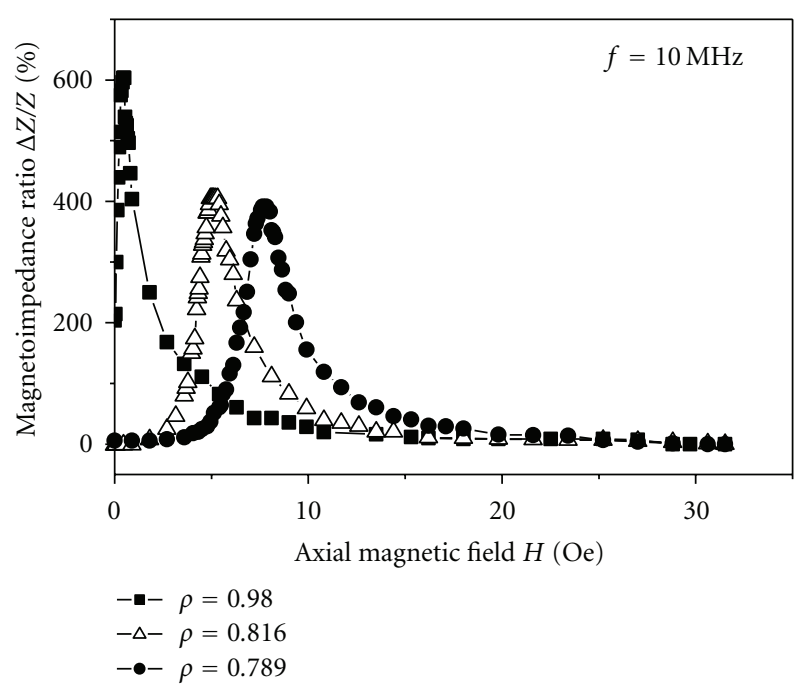

Figure 2: Axial field dependence of $\Delta Z / Z$ at $f=10 \mathrm{MHz}$ and $I=$ $0.75 \mathrm{~mA}$ in microwire with $\rho$ as a parameter.

It has been demonstrated that the spatial magnetization distribution close to the surface is very sensitive to the internal or applied stresses $[11,12]$.

It was shown that the $(\Delta Z / Z)(H)$ dependence is mainly determined by the type of magnetic anisotropy [12]. Circumferential anisotropy leads to the observation of the maximum of the real component of wire impedance (and consequently of the GMI ratio) as a function of the external magnetic field. In the case of axial magnetic anisotropy, the maximum value of the GMI ratio corresponds to zero magnetic field [12], that is, results in monotonic decay of GMI ratio with $H$. Therefore, the important contribution of the nondiagonal components of the permeability tensor is expected for the samples with well-defined maximum in the axial field dependence of GMI ratio [12].

The MOKE hysteresis loop reflects the axial field dependence of circular magnetization (see Figure 3 ) in the outer shell of the wire. The transverse configuration of MOKE has been used. The polarized light of a He-Ne laser was reflected from the microwire to the detector. The beam diameter was $0.8 \mathrm{~mm}$. For the transverse configuration of MOKE, the intensity of the reflected light was proportional to the magnetization, which was perpendicular to the plane of incident light.

Observed MOKE hysteresis loops can be interpreted in the following way: the absence of the circular magnetization under axial magnetic field above $2 \mathrm{Oe}$ reflects the axial alignment of the magnetization in the surface layer at this magnetic field range. The monotonic increase of the magnetization with decreasing the field below $2 \mathrm{Oe}$ is related to the magnetization rotation from the axial to the circular direction. The relatively sharp change of magnetization (at around $\pm 0.7 \mathrm{Oe}$ ) could be attributed to the nucleation of new domains with the opposite circular magnetization (appearance of bamboo-like domain structure), and growth of these new domains through the domain walls propagation, until

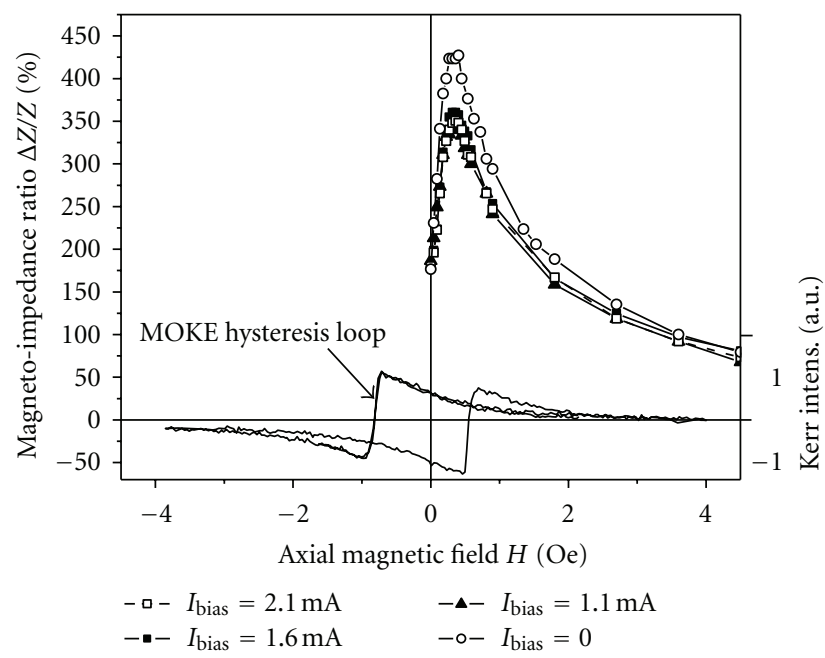

FIGURE 3: $\Delta Z / Z$ and MOKE hysteresis loop of microwire with $\rho=$ 0.98 .

the single circular domain structure with the opposite circular magnetization is appeared. Further increase of $H$ results in the magnetization rotation towards the axial direction.

A correlation has been observed between the $(\Delta Z / Z)(H)$ and the surface axial hysteresis loops: a maximum of GMI ratio occurs approximately at the same axial magnetic field as the sharp change of magnetization on the MOKE loop (see Figure 3).

\section{Effect of the Sample Composition and Geometry on the Bulk Hysteresis Loops}

Hysteresis loops of Co-rich glass-coated microwires with sample composition as the parameter $\left(\mathrm{Co}_{69-x} \mathrm{Mn}_{6+x} \mathrm{Si}_{10} \mathrm{~B}_{15}\right)$ are shown in Figure 4 [3].

As can be observed, hysteresis loops are quite sensible to both parameters chemical composition and sample geometry (Figure 1).

Such strong dependence of the hysteresis loops on these parameters can be related with the magnetoelastic energy. In fact the magnetoelastic energy is given by

$$
K_{\mathrm{me}} \approx 3 / 2 \lambda_{s} \sigma_{i},
$$

where $\lambda_{s}$ is the saturation magnetostriction, and $\sigma_{i}$ are the internal stresses.

The magnetostriction constant depends on the chemical composition achieving nearly zero value in amorphous FeCo- based alloys at about $\% \mathrm{Co} / \% \mathrm{Fe} \approx 70 / 5[4,5]$. On the other hand, the estimated values of the internal stresses in these amorphous microwires arising from the difference in the thermal expansion coefficients of metallic nucleus and glass coating are of the order of $100-1000 \mathrm{MPa}$, depending strongly on the thickness of glass coating and metallic core diameter [13]. It was established that the strength of such internal stresses increase with increasing the glass coating thickness. Such large internal stresses give rise to a drastic change of the magnetoelastic energy, $K_{\text {mei }}$, even for small 


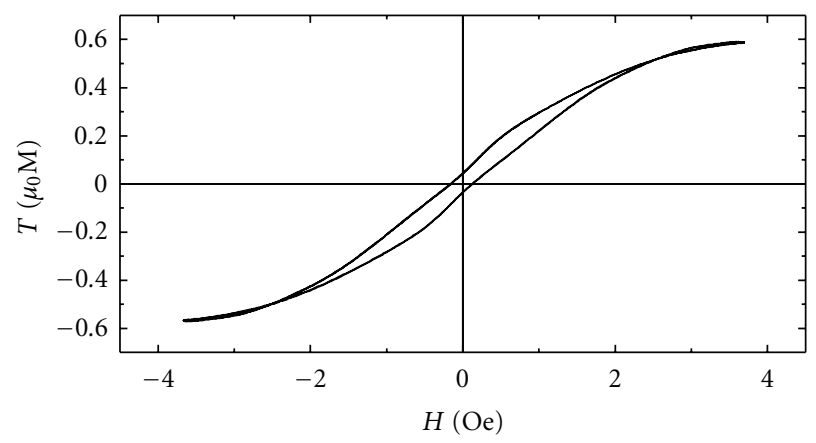

(a)

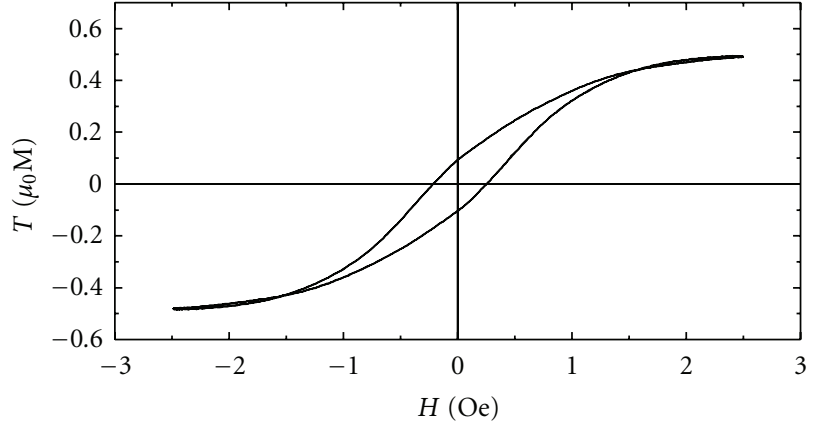

(b)

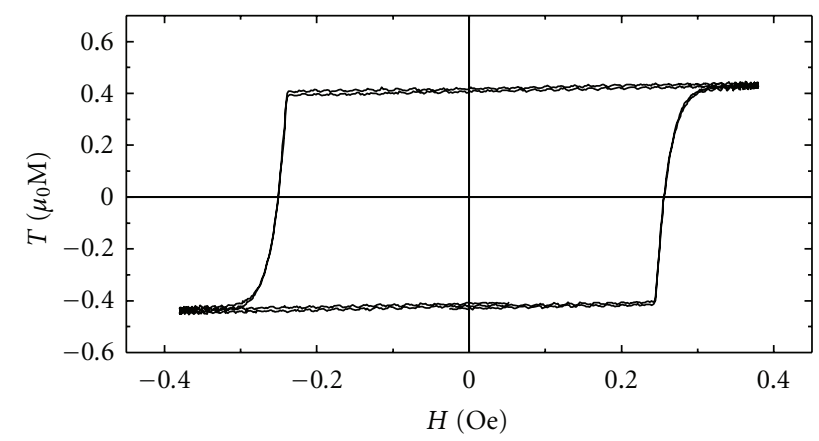

(c)

Figure 4: Hysteresis loops of the $\mathrm{Co}_{69-x} \mathrm{Mn}_{6+x} \mathrm{Si}_{10} \mathrm{~B}_{15}$ microwires. $x=1$ (a); $x=0,5$ (b); $x=0$ (c).

changes of the glass-coating thickness at fixed metallic core diameter. Additionally, such change of the $\rho$-ratio should be related to the change of the magnetostriction constant with applied stress [14]:

$$
\lambda_{s}=\left(\frac{\mu_{0} M_{s}}{3}\right)\left(\frac{d H_{k}}{d \sigma}\right),
$$

where $\mu_{0}$ is the free space permeability equals $4 \pi \times 10^{-7} \mathrm{H} / \mathrm{m}$ and $M_{s}$-the saturation magnetization.

These considerations allow us to predict that any method to change the internal stresses (by using thermal treatment, chemical etching, etc.) can change drastically magnetic anisotropy and consequently the hysteresis loops and the GMI behaviour.

Thermal treatment has been realized either by Joule heating passing along the microwire sample a DC-current of 30 and $40 \mathrm{~mA}$, at various times of this treatment or by conventional furnace annealing. This Joule heating has been performed without additional external field (CA) and under axial applied magnetic field of about 100 Oe (MFA). Electrical contacts were made removing mechanically the glass insulating layer at the sample edges and soldering them with the $\mathrm{Cu}$ cables.

Consequently, the hysteresis loops of $\mathrm{CO}_{69-x} \mathrm{Mn}_{6+x} \mathrm{Si}_{10} \mathrm{~B}_{15}$ $(x=0.5)$ has been changed after MFA treatment (see Figure 5), and the GMI response has been improved.

Similarly, in the case of $\mathrm{Co}_{69-x} \mathrm{Mn}_{6+x} \mathrm{Si}_{10} \mathrm{~B}_{15}(x=0.5)$ microwire, the MFA treatment of $\mathrm{CO}_{67} \mathrm{Fe}_{3.85} \mathrm{Ni}_{1.45} \mathrm{~B}_{11.5} \mathrm{Si}_{14.5} \mathrm{Moo}_{1.7}$ microwire also induces changes in hysteresis (see Figure 6). In this case, the external axial magnetic field applied during the MFA treatment induces axial magnetic anisotropy, like what can be appreciated from Figure 6 .

It is worth mentioning that appearance of Large and single Barkhausen jump takes place under magnetic field above some critical value (denominated as switching field) and also if the sample length is above some critical value denominated also as critical length. The switching field depends on magnetoelastic energy determined by the strength of the internal stresses, applied stresses and magnetostriction constant. The critical length first increases with stress, but then again decreases.

Conventional annealing performed at $T_{\mathrm{ann}}=400^{\circ} \mathrm{C}$ in $\mathrm{Fe}_{74} \mathrm{~B}_{13} \mathrm{Si}_{11} \mathrm{C}_{2}$ does not affect significantly the magnetic properties of studied sample (see Figure 7): some decrease of $H_{c}$ has been observed, while the squared character of the hysteresis loop remains unchanged (Figure $7(\mathrm{~b})$ ). On the other hand, stress annealing, (SA), is performed at the same annealing conditions $\left(T_{\mathrm{ann}}=400^{\circ} \mathrm{C}\right)$ but under applied stress, $\sigma=458 \mathrm{MPa}$, results in drastic changes of the hysteresis loop (see Figure 7(c)): hysteresis loop becomes inclined with a magnetic anisotropy field about $1000 \mathrm{~A} / \mathrm{m}$. A transverse magnetic anisotropy induced by the SA allows us to predict the existence of the magnetoimpedance effect in such samples.

Above-mentioned results allow us to assume that there are few factors which can affect the GMI behaviour of the glass coated microwires such as-following:

(a) metallic nucleus chemical composition;

(b) samples geometry;

(c) thermal treatments. 


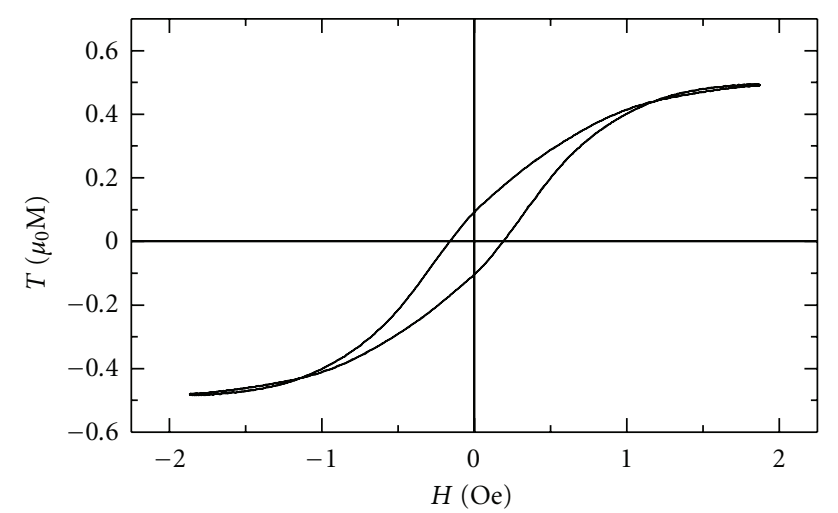

(a)

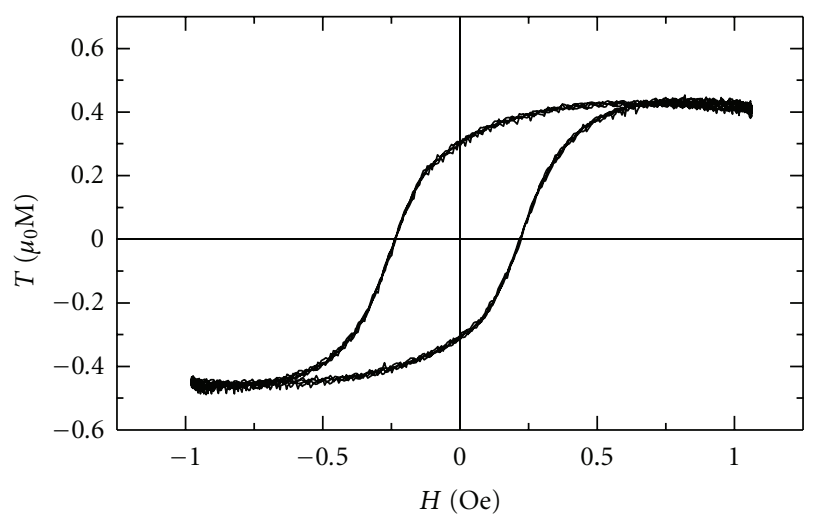

(b)

Figure 5: Effect of the MFA (current annealing under axial applied magnetic field) treatment on hysteresis loops of $\mathrm{CO}_{69-x} \mathrm{Mn}_{6+x} \mathrm{Si}_{10} \mathrm{~B}_{15}$ $(x=0.5)$ microwires: (a) as-cast; (b) MFA.

\section{Effect of the Sample Composition on the Surface Hysteresis Loops}

The Kerr effect experiments have been performed in microwires of nominal composition $\left(\mathrm{Co}_{1-x} \mathrm{Mn}_{x}\right)_{75} \mathrm{Si}_{10} \mathrm{~B}_{15}$ (diameter around $20 \mu \mathrm{m})$ with different content of $\mathrm{Mn}(0.07<x<$ 0.11) [15].

There were four schemes of the experiments, depending on the combination of magnetic field combination and the type of Kerr effect:

(1) transverse Kerr effect at sweeping of circular field (+ axial bias field);

(2) longitudinal Kerr effect at sweeping of circular field (+ axial bias field);

(3) transverse Kerr effect at sweeping of axial field (+ circular bias field);

(4) longitudinal Kerr effect at sweeping of axial field (+ circular bias field).

The obtained Kerr effect loops show different shape, which can be attributed to different type of domain structure and difference in the magnetization reversal process (Figure 8).

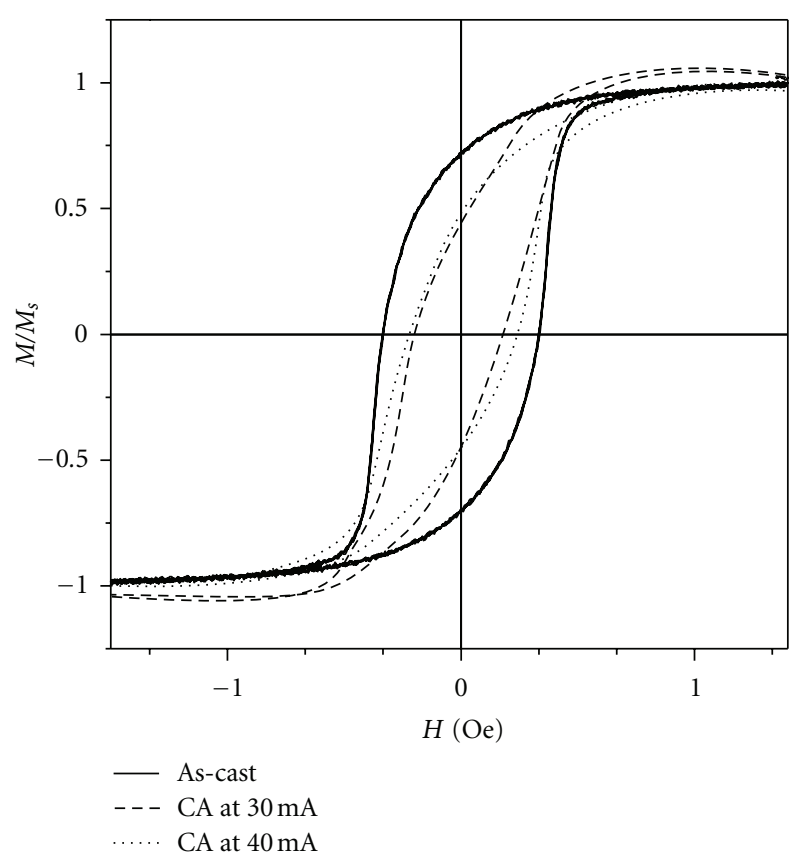

(a)

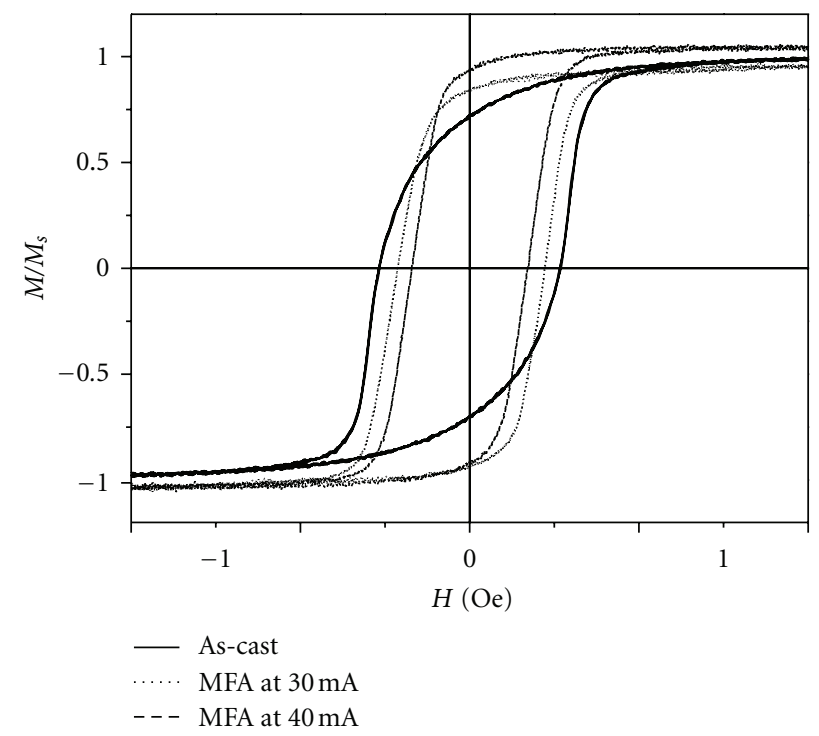

(b)

FIGURE 6: Effect of CA (current annealing without additional external magnetic field) (a) and MFA (b) treatments on bulk hysteresis loops of $\mathrm{Co}_{67} \mathrm{Fe}_{3.85} \mathrm{Ni}_{1.45} \mathrm{~B}_{11.5} \mathrm{Si}_{14.5} \mathrm{Mo}_{1.7}$ microwires.

The transverse and longitudinal Kerr effect loops obtained in ac circular field for the microwire with $x=0.07$ are presented in the Figures 8(a) and 8(b). From the analysis of these curves, it is possible to conclude that jumps of circular magnetization take place. The rectangular shape of transverse curve and the peaks in the longitudinal curve are related to quick rotation of magnetization in circular surface domain similar to large Barkhausen jump.

For the microwire with content of $\operatorname{Mn} x=0.11$, the rectangular shape of the magnetization reversal curve was found 


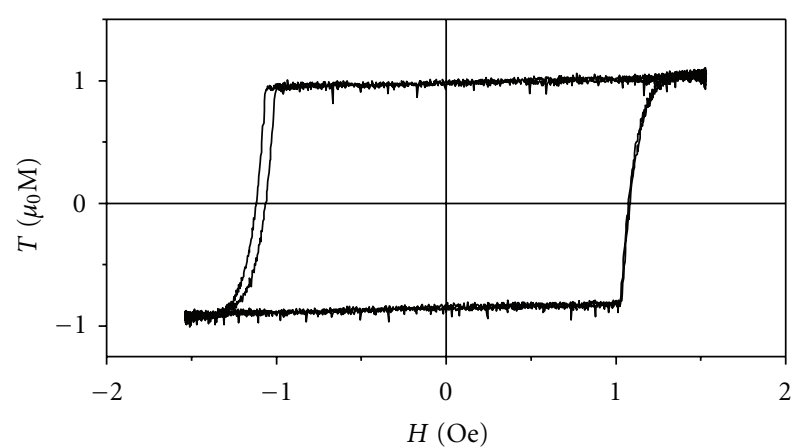

(a)

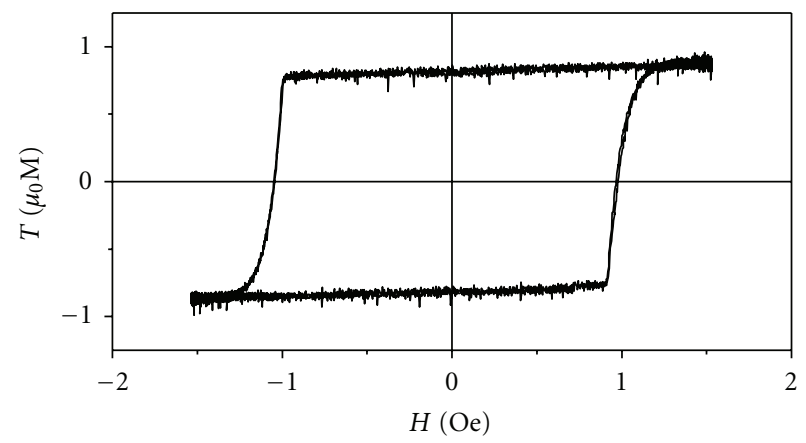

(b)

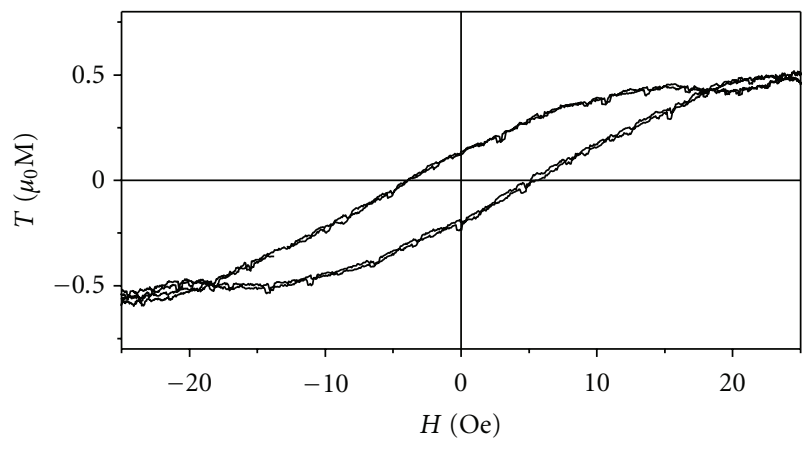

(c)

FIgURE 7: Effect of CA and SA (stress annealing) on bulk hysteresis loops of $\mathrm{Fe}_{74} \mathrm{~B}_{13} \mathrm{Si}_{11} \mathrm{C}_{2}$ microwire: (a) as cast; (b) conventional annealing; (c) stress annealing.

in $a c$ axial field (Figures 8(c) and 8(d)). The longitudinal Kerr loop reflects the change of the axial projection of the magnetization inside the surface area of the wire. Therefore, the sharp change of longitudinal Kerr effect (Figure $8(\mathrm{c})$ ) could be attributed to a quick change of the axial magnetization.

The transformation of transverse loops in presence of $d c$ circular field could clarify the details of the magnetization process (Figure $8(d)$ ). The absence of signal when the axial field is zero can be explained taking into account the absence of a circular projection during magnetization reversal. That is, the magnetization reversal occurs only by domain walls motion between the axial domains. Under the application of the $d c$ circular field, the magnetization inside the domain deviates from the axial direction, the transverse projection appears, and the transverse Kerr loops are observed. In this way, the magnetization reversal process can occur by the domain wall motion owing to the rotation of the magnetization.

The magnetization reversal for the microwire with $x=$ 0.09 is close to that of the microwire $x=0.11$, but some peculiarities are observed (Figures $8(\mathrm{e})$ and $8(\mathrm{f})$ ). The longitudinal loop obtained in ac axial field presents a rectangular shape related to the change of the axial magnetization similarly to the case of $x=0.11$. At the same time, the transverse loop has the shape, which can be attributed to successive rotation of magnetization and nucleation of new domains.

The modification of the domain structure of glasscoated amorphous microwires $\left(\mathrm{Co}_{1-x} \mathrm{Mn}_{x}\right)_{75} \mathrm{Si}_{10} \mathrm{~B}_{15}$ could be ascribed to the change of the value and the sign of the magnetostriction constant. As was shown in [16], the $\lambda_{\mathrm{S}}$ is positive for the wires with $x \geq 0.1$ and negative for the wires with $x<0.1$. Taking into account that the circular magnetization process in the outer shell is attributed to the negative sign of the magnetostriction, and axial magnetization in outer shell is attributed to positive magnetostriction, our magneto-optical investigation for the wire with $x=0.07$ and for $x=0.11$ is in agreement with independent examination of the magnetostriction constant value. It is necessary to note that the quick magnetization reversal discovered in wire with $x=0.07$ can be considered like large Barkhausen jump in the circular magnetic structure. This effect was observed in Co-rich amorphous wire when an electric current is flowing along the wire to produce a circular magnetic field.

In spite of that for the microwire with $x=0.09 \lambda_{\mathrm{S}}$ is negative, the axial domain structure exists in this wire. Significant contribution of rotation of the magnetization is found in this wire together with domain nucleation, which suggests that this wire occupies intermediate place between wires with $x=0.07$ and $x=0.11$.

Therefore, Co-rich microwires with different content in Mn demonstrate the variety of the shape of magnetization reversal loop, which similarly can be attributed to the change of sigh and value of the magnetostriction. The rectangular shape of the hysteresis loop in circular magnetic field of the microwire with $x=0.07$ can be interpreted by considering that the magnetization process in the outer shell takes place by large Barkhausen jumps of circular domain structure, while the rectangular shape of the Kerr hysteresis loop in axial magnetic field of the microwire with $x=0.11$ could be connected to large Barkhausen jumps in the axial domain structure in the outer shell.

\section{Surface Circular Bistability}

Glass-covered microwires of nominal composition $\mathrm{Co}_{67} \mathrm{Fe}_{3.85} \mathrm{Ni}_{1.45} \mathrm{~B}_{11.5} \mathrm{Si}_{14.5} \mathrm{Mo}_{1.7}$ (metallic nucleus radius $R=11.2 \mu \mathrm{m}$, glass coating thickness $T=0.2 \mu \mathrm{m}$ ) have been studied using transverse MOKE in axial and circular magnetic field [17].

Figure 9 presents the transverse Kerr effect dependence on the ac electric current $I$ flowing with the frequency of 


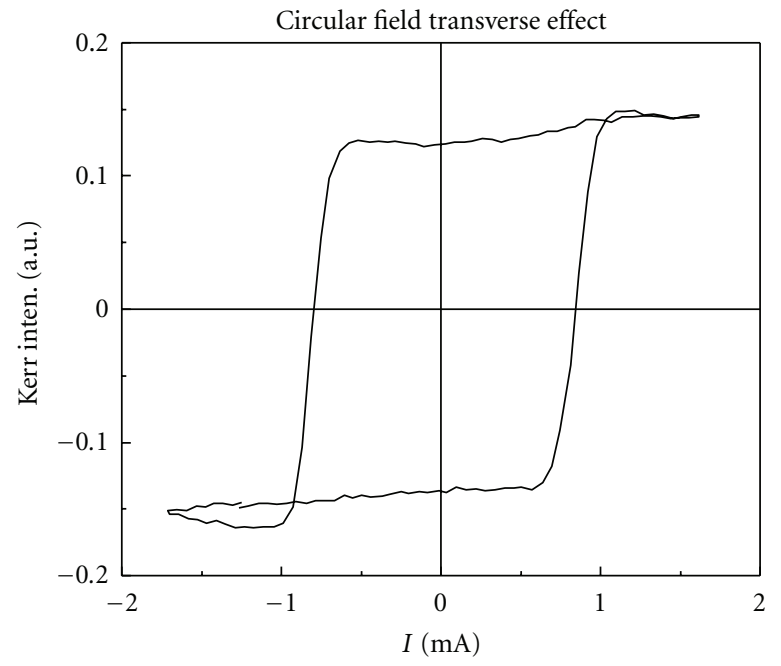

(a)

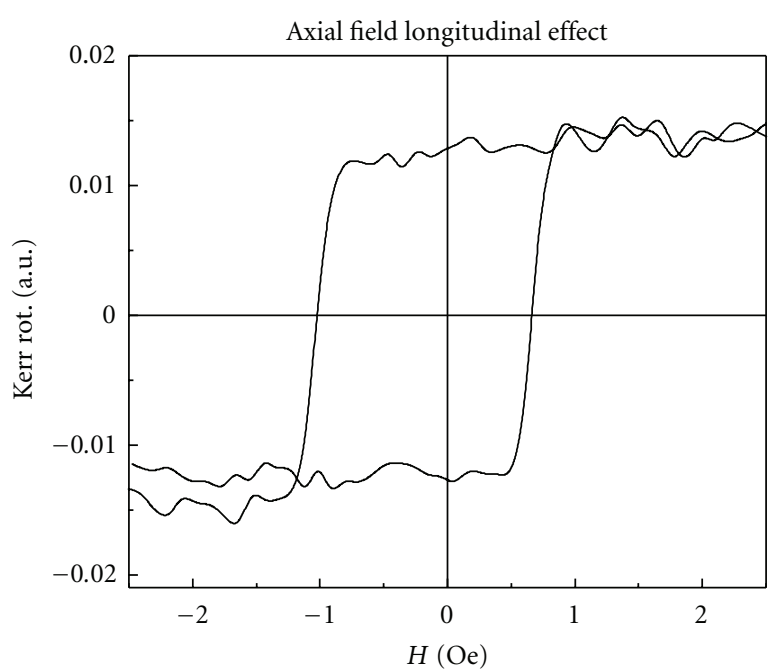

(c)

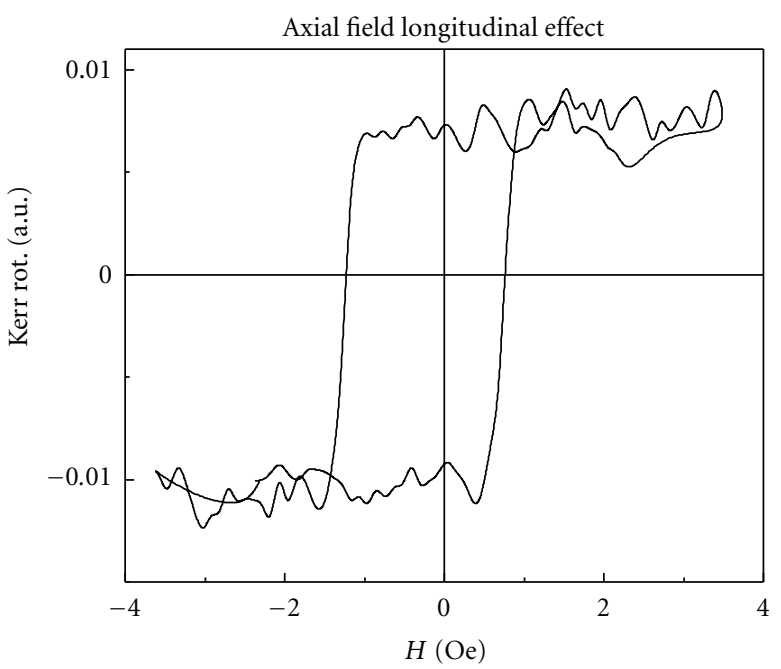

(e)

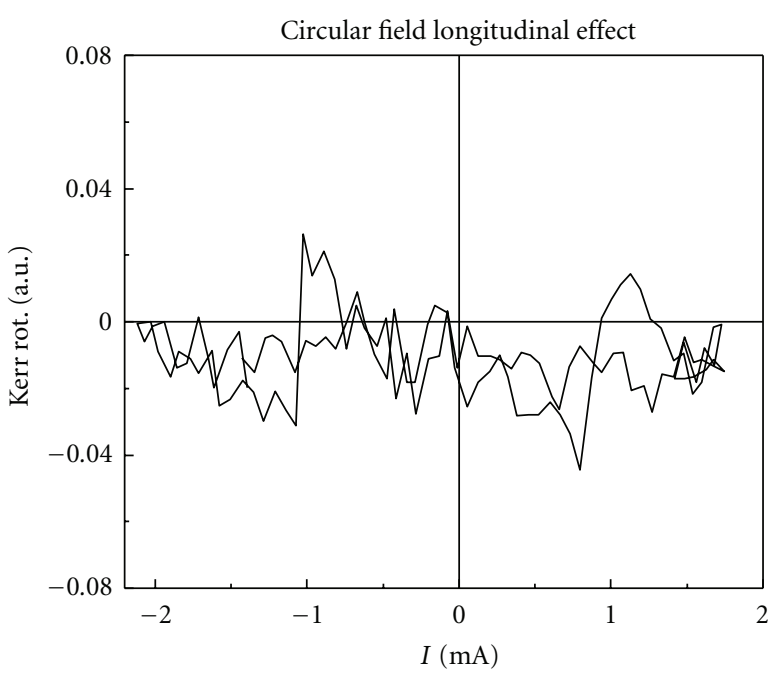

(b)

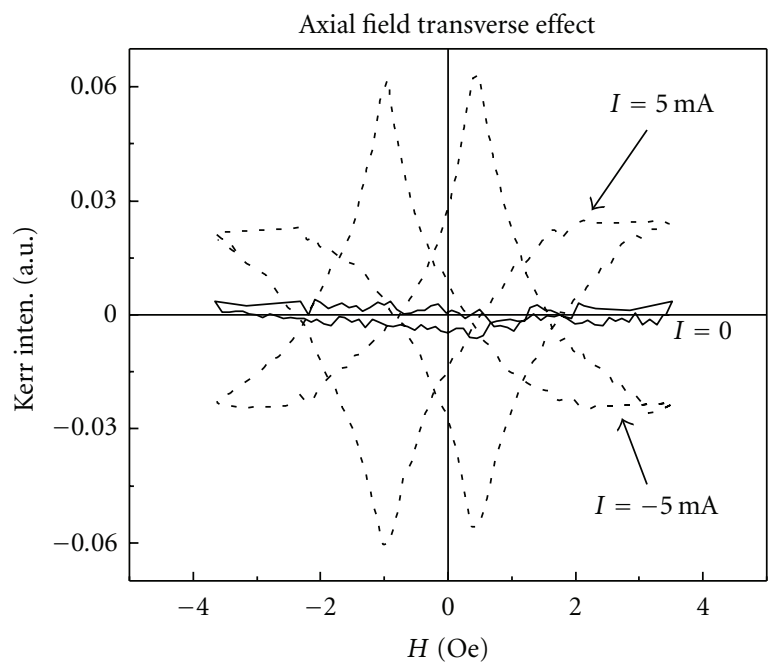

(d)

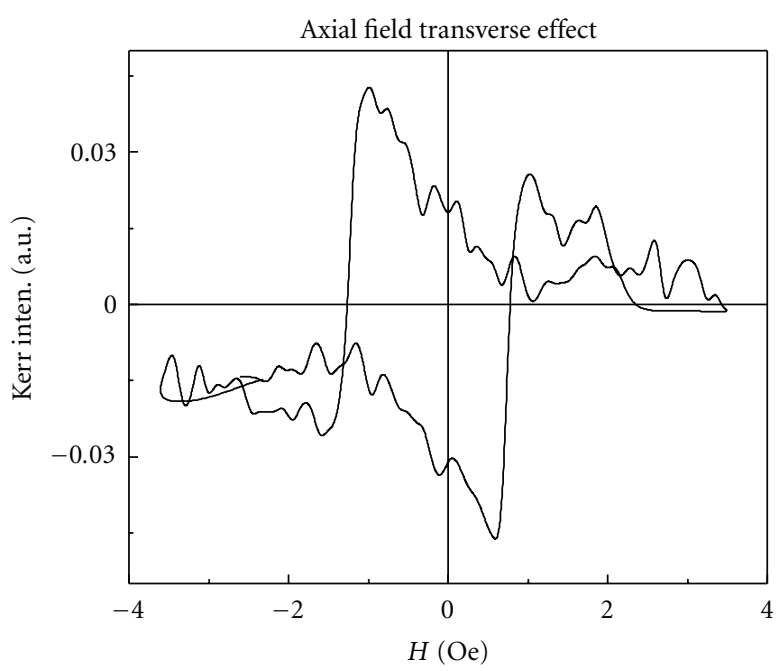

(f)

Figure 8: Longitudinal and transverse Kerr effect loops of the glass-coated $\left(\mathrm{Co}_{1-x} \mathrm{Mn}_{x}\right)_{75} \mathrm{Si}_{10} \mathrm{~B}_{15}$ amorphous microwires for different Mn content: (a)-(b) $x=0.07$; (c)-(d) $x=0.11$; (e)-(f) $x=0.09$. 


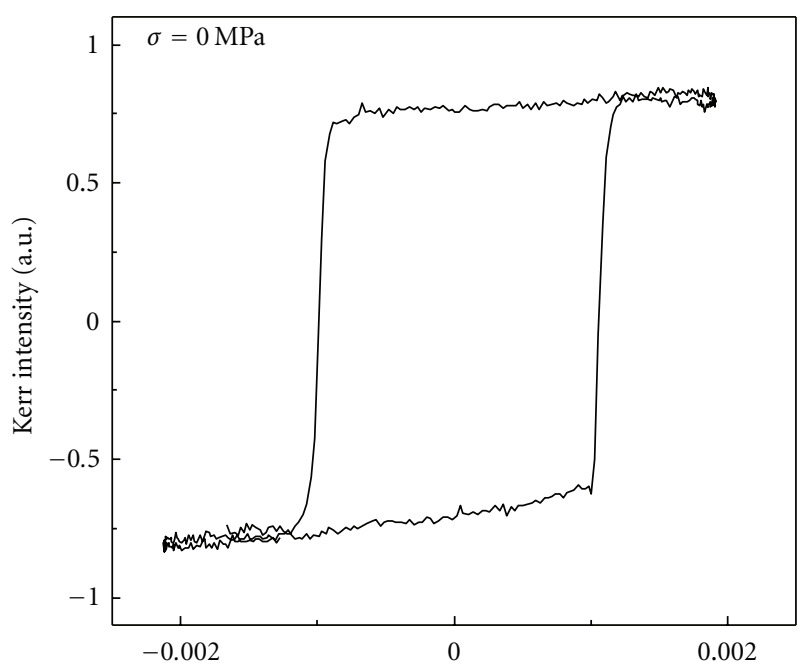

(a)

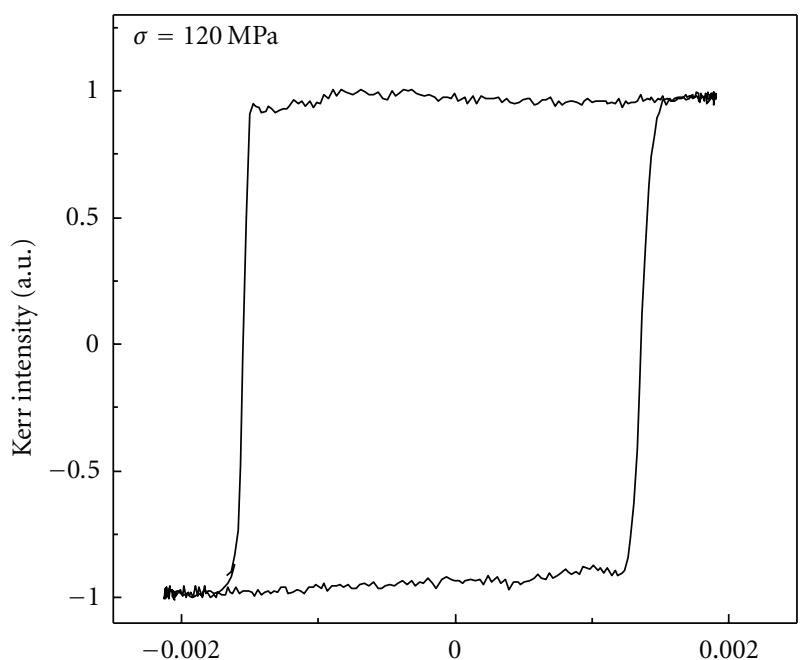

(b)

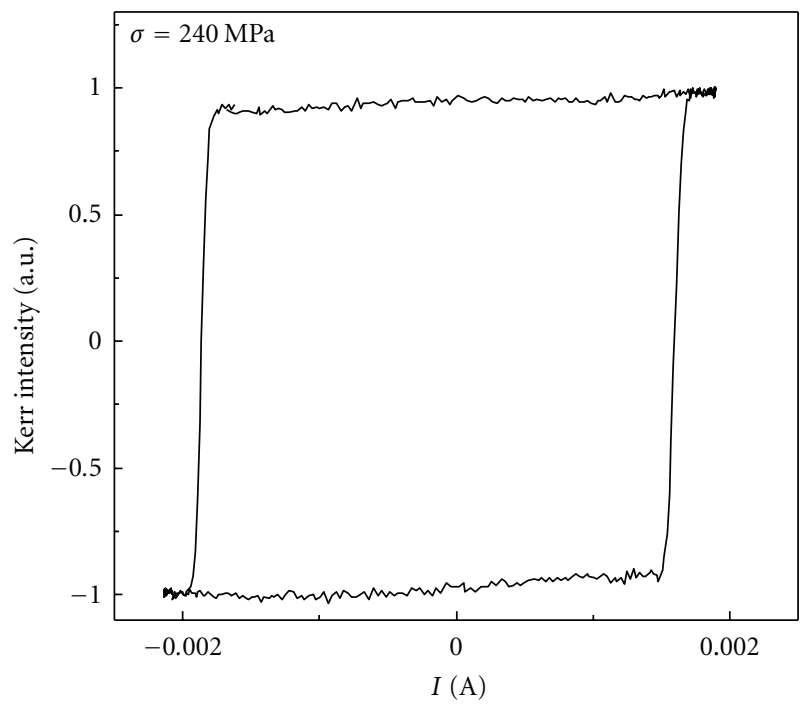

(c)

Figure 9: Transverse Kerr effect dependence on ac electric current flowing with the frequency of $50 \mathrm{~Hz}$ through the wire for different tensile stresses: (a) $0 \mathrm{MPa}$; (b) $120 \mathrm{MPa}$; (c) $240 \mathrm{MPa}$.

$50 \mathrm{~Hz}$ along the sample with an external tensile stress $\sigma$ as a parameter. The shape of hysteresis loop is perfectly rectangular with sharp vertical areas associated with quick enough reversal of circular magnetization in the absence of external tensile stress (Figure 9(a)). This reversal is a realization of circular magnetic bistability in the form of large Barkhausen jumps between two states with opposite directions of circular magnetization. The external tensile stress causes the change of switching field, $H_{\mathrm{SW}}$, (associated with the switching current).

The change of the $I_{R} / I_{S}$ ratio under the tensile stress is also observed $\left(I_{R}\right.$ is the intensity of the Kerr signal in the remanent state, and $I_{\mathrm{S}}$ is the intensity of the Kerr signal in the saturation state). The Kerr intensity is proportional to transverse magnetization $\mathrm{M}$ in the surface area of the wire. There- fore, it is possible to consider that $I_{\mathrm{R}} / I_{\mathrm{S}}=M_{\mathrm{R}} / M_{\mathrm{S}}$, where $M_{\mathrm{R}}$ is the transverse saturation magnetization, and $M_{\mathrm{S}}$ is the transverse remanent magnetization.

The circular magnetic bistability is related to the magnetoelastic anisotropy in the circular direction, as the classical longitudinal magnetic bistability $[18,19]$. The circular magnetoelastic anisotropy results in the appearance of circular monodomain structure in the outer shell. It is known that in negative magnetostrictive wires the axial tensile stress produces circular anisotropy in the outer shell. In the presented experiments, this effect is reflected in the increase of circular remanence magnetization and in the increase of the squarness of the hysteresis loops in presence of tensile stress.

The tensile stress-induced increase of circular switching field could be explained taking into account the strong 


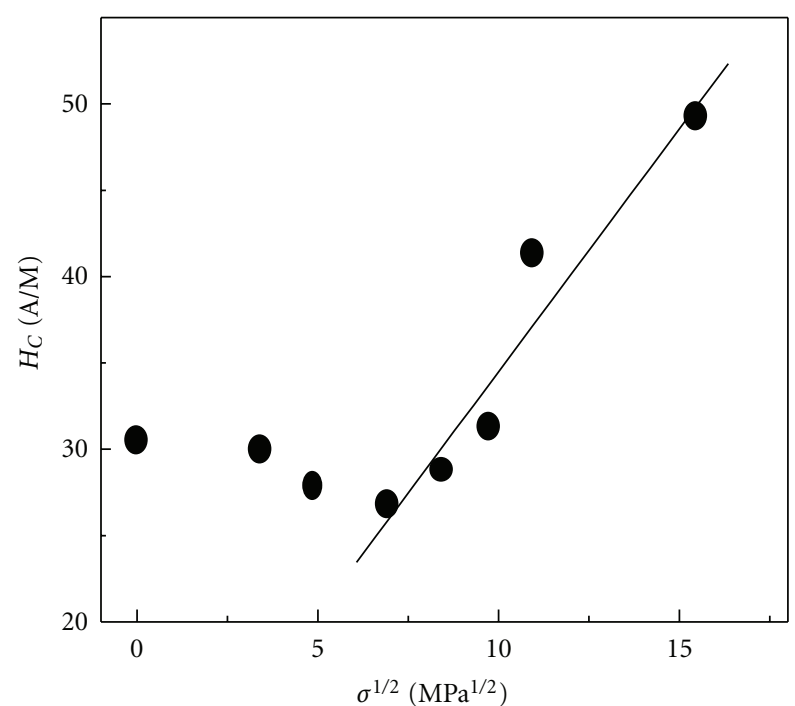

FIGURE 10: The circular switching field as a function of the square root external tensile stress.

influence of the magnetostriction on the domain structure in the amorphous wires. The circular bistability effect is related to formation of circular domain walls. The circular switching field should be proportional to the energy $\gamma$ required to form the circular domain wall. The circular switching field is related to the magnetoelastic anisotropy as given by $[20,21]$ :

$$
H_{\mathrm{SW}} \infty \gamma \infty\left[\frac{3}{2 A \lambda_{\mathrm{S}}\left(\sigma_{\mathrm{a}}+\sigma_{\mathrm{r}}\right)}\right]^{1 / 2},
$$

where $A$ is the exchange energy constant, $\sigma_{\mathrm{a}}$ is applied tensile stress, $\sigma_{\mathrm{r}}$ is the internal tensile stress, and $\lambda_{\mathrm{S}}$ is magnetostrictive constant. The switching field must be proportional to $\sigma_{\mathrm{a}}^{1 / 2}$ for the applied stress $\sigma_{\mathrm{a}}$ larger than the internal stress $\sigma_{\mathrm{r}}$, that is, when the remanence has reached the saturation value. The magnetostriction constant of this microwire is negative, so it is expected that the volume of outer shell will increase with the circular anisotropy. Due to the increase of circular anisotropy region with the stress at the expense of the reduction of the axial anisotropy volume, the circular remanence increases as a function of the applied stress. For high value of $\sigma_{\mathrm{a}}$, there will be no contributions from the axial volume, and the circular remanence reaches its saturation value.

The experimental $H_{\mathrm{SW}}$ dependence on the tensile stress has been plotted as a function of the square root applied stress $\sigma_{\mathrm{a}}$ (Figure 10). Good fitting of the experimental points by the linear dependence take place for the $\sigma_{\mathrm{a}}>50 \mathrm{MPa}$, when the circular remanence reaches the saturation. Consequently, we can estimate the value of the $\sigma_{\mathrm{r}}$ internal stress as about $50 \mathrm{MPa}$. Such small value of the internal stress seems to be acceptable taking into account the extremely small value of glass coating in the studied wire. The observed decrease of $H_{\mathrm{SW}}$ for low-applied tensile stress is probably a result of the tensor character of internal stresses with an important contribution of radial and circular stresses components.

\section{Tensile Stress Influence on Surface Magnetization Reversal}

Figure 11 shows the stress-induced changes of surface hysteresis loop in the microwire of nominal composition $\mathrm{Co}_{67} \mathrm{Fe}_{3.85} \mathrm{Ni}_{1.45} \mathrm{~B}_{11.5} \mathrm{Si}_{14.5} \mathrm{Mo}_{1.7}$ (metallic nucleus radius $R=$ $11.2 \mu \mathrm{m}$, glass coating thickness $T=3 \mu \mathrm{m}$ ). The experimental results have been obtained using MOKE magnetometer. The monotonic hysteresis loop is observed with $\sigma=0$ (Figure 11(a)). The sharp jumps of magneto-optical signal arising in the presence of the tensile stress (Figure 11(c)) should be attributed to circular bistability. Another significant feature is the increase of the value of the circular switching field with increasing tensile stress. This indicates the change of coercivity of circular domain structure under the tensile stress.

The transverse Kerr effect experiments in ac axial field (Figures 11(d)-11(f)) give additional information about the formation of circular domain structure and open new details of the magnetization reversal process. It is worth mentioning that these three graphs were plotted in the same scale of Kerr intensity. Figures 11(e) and 11(f) represent the typical behavior of circular domain structure in axial field. The monotonic increase of the Kerr signal with increase of field could be related to the rotation of magnetization from axial to circular direction. Sharp jump of the signal, which is followed by the change of the sign of the signal, is associated with nucleation of new circular domain with the opposite direction of the magnetization. The last part of the loop is the monotonic decrease of the signal reflected the magnetization rotation from circular into the axial direction.

The transverse hysteresis loop with perfectly squared shape appears step by step under the applied tensile stress (Figure 11). In the intermediate stage (tensile stress of $30 \mathrm{MPa}$ ), the Kerr effect curve contains monotonic parts and jumps (Figure 11(b)), which could be associated with the rotation of magnetization and the motion of domain walls between circular domains. Also the minor loop is observed at this tensile stress.

The existence of such hysteresis loop permits us to suppose the existence of a multidomain circular bamboo structure in the studied microwires. The Kerr microscopy experiments confirm this supposition.

The results obtained have been explained taking into account the strong correlation between the magnetostriction and the domain structure in the amorphous microwires. This correlation is based on the competition between the magnetostatic and magnetoelastic energies. The magnetostatic energy depends on the demagnetizing field. We consider, in our experimental results, the supposition that one of the reasons for the observed transformation of the hysteresis loops is the change of the sign of the magnetostriction constant. The large Barkhausen jump of the axial magnetization in the outer shell could be attributed to positive value of magnetostrictive constant, while the large Barkhausen jump of the circular magnetization in the outer shell for could be attributed to negative value of magnetostrictive constant.

Considering that $\lambda_{S}(\sigma)=0$ for tensile stress of about $30 \mathrm{MPa}$, we could estimate the value of phenomenological 


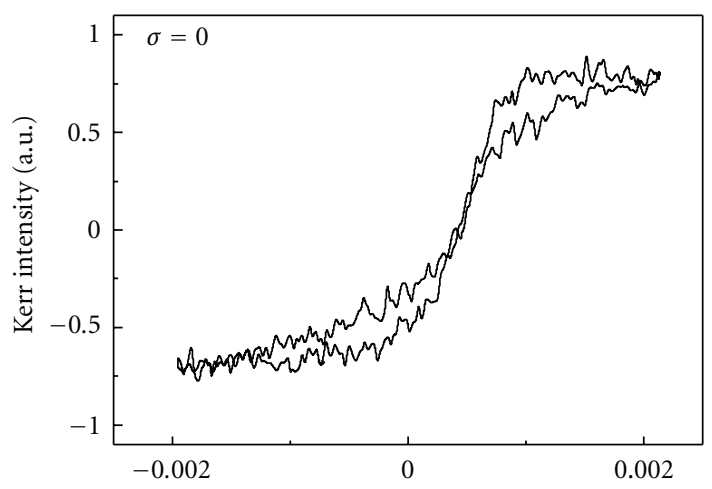

(a)

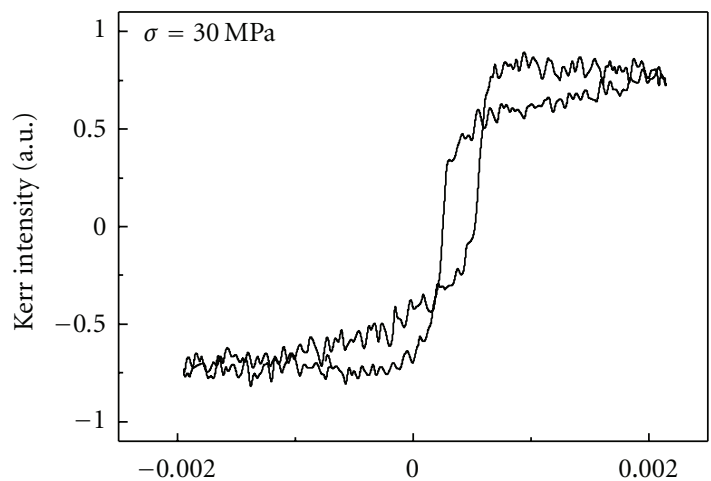

(b)

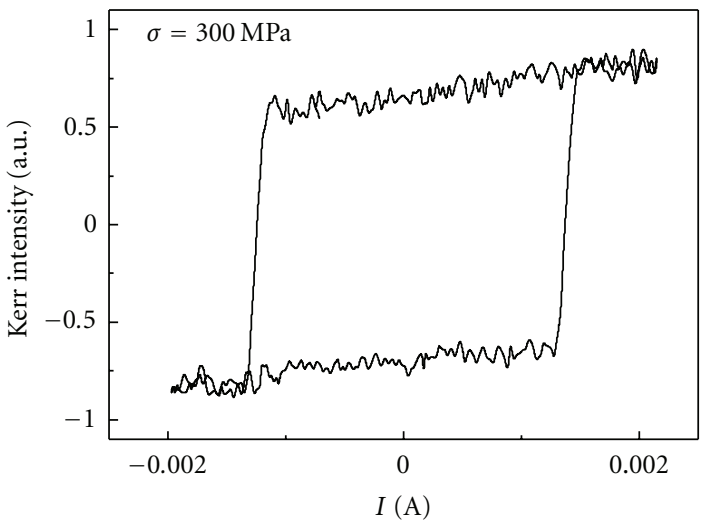

(c)

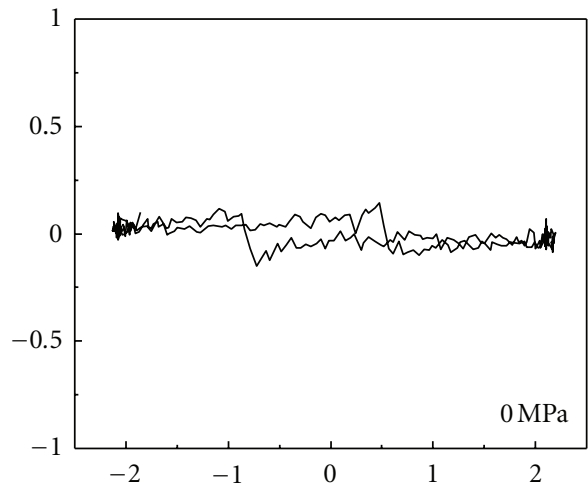

(d)

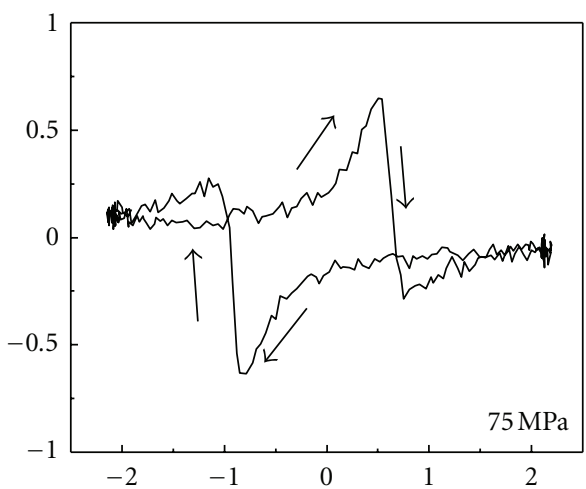

(e)

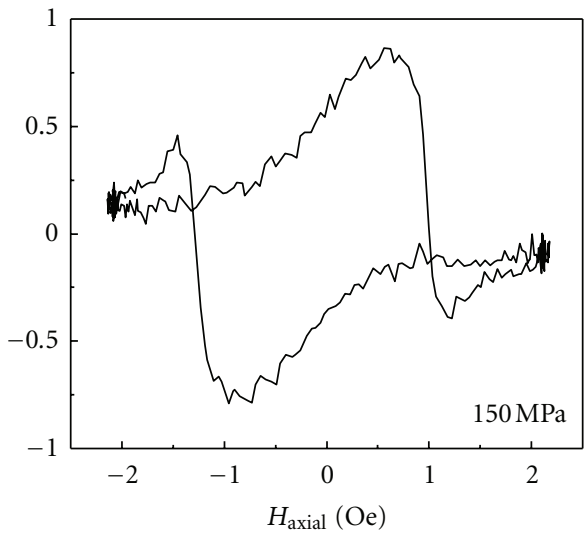

(f)

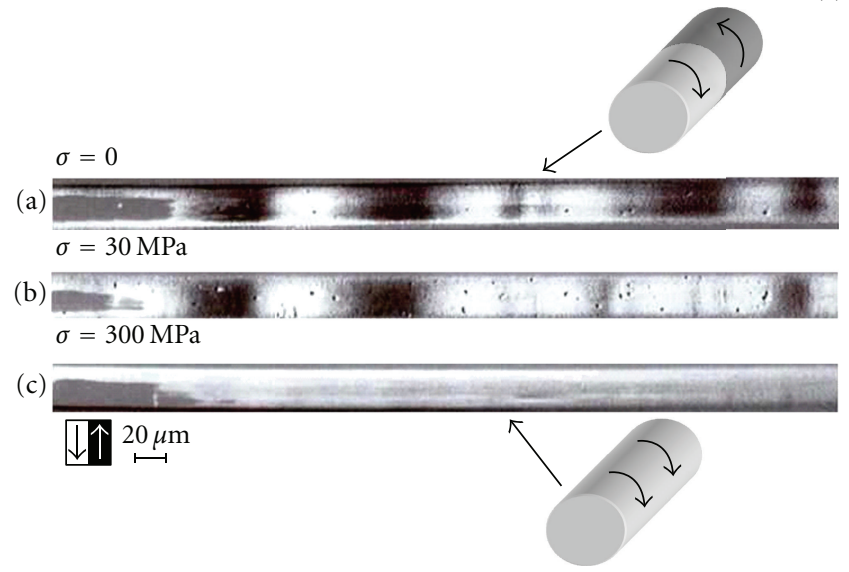

FIgURE 11: Transverse Kerr-effect dependencies: (a)-(c) on the electric current flowing through the wire in the presence of tensile stress; (d)-(f) on the axial magnetic field in the presence of tensile stress. Inset: circular domain structure in presence of external tensile stress in microwire. 


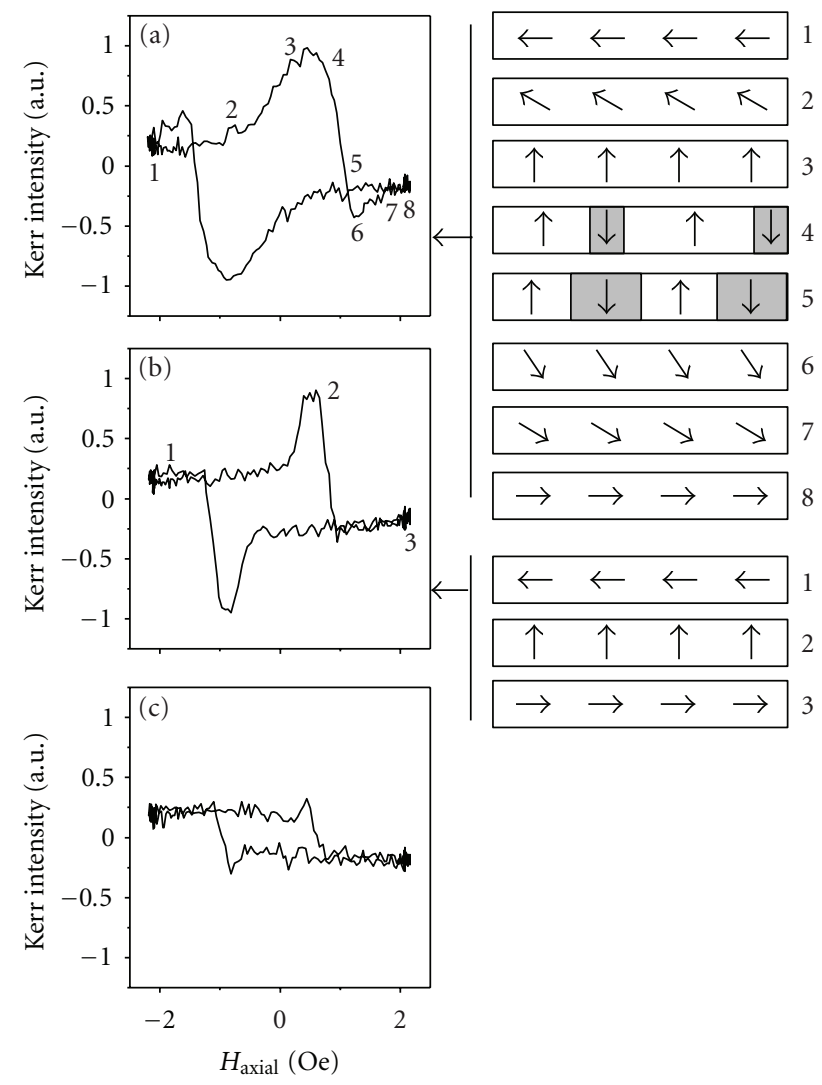

Figure 12: Transverse Kerr effect dependence on the axial magnetic field under the presence of HF electric current (4 kHz) (a)—(c): (a) $0 \mathrm{~mA}$, (b) $0.76 \mathrm{~mA}$, and (c) $1.35 \mathrm{~mA}$.

A constant for glass-covered wires to be about $3 \times 10^{-9} \mathrm{MPa}$. This value differs from the value of $A$ for conventional wires and ribbons $\left(10^{-10} \mathrm{MPa}\right)$. The possible reason for this difference is additional internal stress introduced by glass coating.

The increase of the tensile stress causes the increase of the value of the circular projection of the magnetization in the outer shell of the microwire, which reflects on the coercive properties of the domain structure. The change of the sign of the magnetostriction constant and its small value are crucial for the observation of changes of the type of the domain structure and for its high sensitivity to external stresses.

For the analysis of our results, we used the core-shell model developed for Co-rich amorphous wires of a finite length [22]. The demagnetizing field of surface magnetic charges can arise near the wire ends for the case of finite length wires. The magnetostatic energy of the magnetic charges could be reduced by twisting, of magnetization near the wire ends. Due to this twisting the intermediate area with circular and axial projections of magnetization could exist between the inner core and the outer shell. Taking into account the strong correlation between the magnetization in the inner core and the outer shell the appearance of domain walls between circular domains could be energetically favorable in order to diminish the magnetostatic energy in whole volume of the wire.

The stability of multidomain circular structure in the nearly zero magnetostrictive composition could be reasonable when the external stresses are small enough because of the competition between the magnetostatic and the magnetoelastic energy at the condition, when the magnetoelastic energy is low enough. The increase of the external tensile stress makes the multidomain structure nonstable, due to the increase of the domain wall energy. Applying additional external stress, we increase the magnetoelastic energy and, consequently, the domain wall energy. At such conditions the bamboo domain structure disappears, and single circular domain state is observed (see domain images in Figure 11).

\section{Influence of High-Frequency Electric Current on Surface Magnetization Reversal}

Figure 12 shows the influence of HF electric current $(f=$ $4 \mathrm{kHz}$ ) flowing along the microwire on the MOKE dependence on the axial magnetic field in the microwire of nominal composition $\mathrm{Co}_{67} \mathrm{Fe}_{3.85} \mathrm{Ni}_{1.45} \mathrm{~B}_{11.5} \mathrm{Si}_{14.5} \mathrm{Mo}_{1.7}$ (metallic nucleus radius $R=8.6 \mu \mathrm{m}$, glass coating thickness $T=$ $0.6 \mu \mathrm{m})$.

The value of the circular magnetic field on the surface of the wire could be obtained using the formula for circumferential field $H_{\text {circ }}=I r / 2 \pi R^{2}$ (for $r=R$ ). The value of $H_{\text {circ }}$ is $0.3 \mathrm{Oe}$ for the value of the ac electric current of $1.35 \mathrm{~mA}$. The experimental setup was tuned in such a way, that the high-frequency signal has been cut by the special filter and therefore we observed only the low-frequency changes of the circular magnetization. Our experiments show that the 
electric current of such amplitude does not produce essential Joule heating.

The insets show how the magnetization in the surface area of the microwire changes during the magnetization reversal. The magnetization reversal in the absence of HF current is demonstrated in Figure 12(a). As mentioned above, at the first stage of the surface magnetization reversal, when the external axial magnetic field $H_{\mathrm{Ax}}$ increases, a rotation of the magnetization from axial to circular direction in the outer shell of the wire is observed (schematic pictures (1)-(3)). At the second stage, the jump of the circular magnetization takes place (schematic pictures (4)-(6)). During this jump, the direction of the circular magnetization reverses (picture (6)). This jump is related to the circular magnetic bistability effect. At the third stage, the magnetization rotation from the circular to the axial direction is observed (pictures (6)-(8)).

The HF current causes the transformation of axial hysteresis loop (Figures 12(b)-12(c)). A decrease of the coercive field, $H_{\mathrm{C}-\mathrm{AX}}$ and $I_{\mathrm{MAX}}$ ( $I_{\mathrm{MAX}}$ is the maximum value of the intensity of the Kerr signal during the magnetization reversal in axial magnetic field) takes place. The coercive field $H_{\mathrm{C}-\mathrm{AX}}$ should be considered as the field, at which the drastic change of the circular magnetization starts in the presence of the axial magnetic field.

The Kerr effect loops presented in the Figure 12 demonstrate the change of the mechanism of the magnetization reversal that is reflected in the change of the shape of the hysteresis curve. The sharp jump (Figure 12(c), pictures (1)-(2)) replaces the monotonic change of the Kerr signal on the first stage of the magnetization reversal (Figures 12(a), pictures (1)-(3)). The HF circular magnetic field induces the jump of magnetization from the axial direction (Figure 12(c), picture (1)) to the circular direction (Figure 12(c), picture (2)) suppressing the process of the rotation of the magnetization.

The HF current has the opposite effect on the surface magnetization reversal as-compared with the tensile stress influence: the tensile stress causes the increase of circular magnetization, and the HF current causes the decrease of circular magnetization. It is interesting to compare two intermediate curves presented in the Figures 11(e) and 12(b). Being quite similar, these hysteresis loops have a difference in the magnetization reversal process. The question is about the presence (Figure 11(e) or absence (Figure 12(b)) of the small peak. This difference confirms that the HF electric current not only suppresses the circular magnetization but also changes the mechanism of the surface magnetization reversal.

\section{Surface and Bulk Magnetic Hysteresis Loops of Co-Rich Microwires with Helical Anisotropy}

Two microwires of nominal composition $\mathrm{Co}_{69.5} \mathrm{Fe}_{3.9} \mathrm{Ni}_{1} \mathrm{~B}_{11.8} \mathrm{Si}_{10.8} \mathrm{Mo}_{2}$ and with different geometric ratio $\rho$ of metallic nucleus diameter $d$ to total microwire diameter $D, \rho=0.785$ (metallic nucleus diameter $d=19 \mu \mathrm{m}$ ) and $\rho=0.885$ (metallic nucleus diameter $d=20 \mu \mathrm{m}$ ) have been studied by transversal MOKE and fluxmetric methods [23].

Experimental Kerr effect hysteresis loops obtained in the axial magnetic field are presented in the Figures 13(a) and 13(b). The surface magnetization reversal in Co-rich amorphous microwires in presence of an axial magnetic field consists of a rotation of the magnetization and a jump of the circular magnetization. For the wire with $\rho=0.785$, the magnetization reversal consists mainly of the rotation of the magnetization, and the jump of the magnetization is quite small, while for the wire with $\rho=0.885$ the jump is large.

The bulk hysteresis loops obtained for the two studied wires in axial magnetic field are presented in Figures 14(a) and $14(\mathrm{~b})$. Just as the experimental curves presented in Figure 13, these loops also have the parts related to the rotation of the magnetization and jumps of the magnetization.

The transverse Kerr effect curves and the conventional curves reflect the change of the circular and axial projection of the magnetization, respectively. Therefore, they could be considered as complement date. From another side, the Kerr effect loops contain information about the surface magnetization reversal when the conventional loops present the magnetization reversal of the whole volume of the wire. Consequently, this complement could not be considered as a complete one.

We believe that the observed difference in surface and bulk hysteresis curves is related to the existence of helical magnetic structure in the studied wires. Generally, the magnetic structure of Co-based amorphous wires was considered in frame of the core-shell model: a Co-based amorphous wire has an inner core with the longitudinal easy magnetization axis and an outer shell with the circular or helical anisotropy [24]. In the glass-covered microwires, the helical magnetic anisotropy originates from the magnetoelastic anisotropy associated with the internal stresses. The internal stresses are produced due to the contraction of the metal and glass having different thermal expansion coefficients [13].

The calculation of the hysteresis loops has been performed taking into account the existence of a helical magnetic anisotropy in the wire. The expression of the energy of the system has the form:

$$
\begin{aligned}
U & =-K_{U} \cos ^{2}(\theta-\varphi)-h \cdot m \\
& =-K_{U} \cos ^{2}(\theta-\varphi)-h_{\text {axial }} \cos (\theta),
\end{aligned}
$$

where $h_{\text {axial }}$ is the applied magnetic field, $K_{U}$ is the uniaxial anisotropy constant, $m$ is the saturation magnetization, $\varphi$ is the angle between the anisotropy axis, and the wire axis and $\theta$ is the angle between the magnetic moment and the wire axis.

The result of the numerical analysis of (5) is presented in Figure 15. There is the calculated dependence of the jump of the circular magnetization $\Delta M_{\mathrm{CIRC}}$ (Figure $13(\mathrm{~d})$ ) on the angle of helical anisotropy. This dependence has a maximum for the angle of $62^{\circ}$. The maximum is not sharp, but for the angles close to $0^{\circ}$ and $90^{\circ}$ the change of the $\Delta M_{\mathrm{CIRC}}$ is strong enough. For the angles close to $90^{\circ}$, the magnetization reversal is determined mainly by a fluent rotation of the magnetization, and this rotation continues after the moment when the magnetization reaches the exact circular 


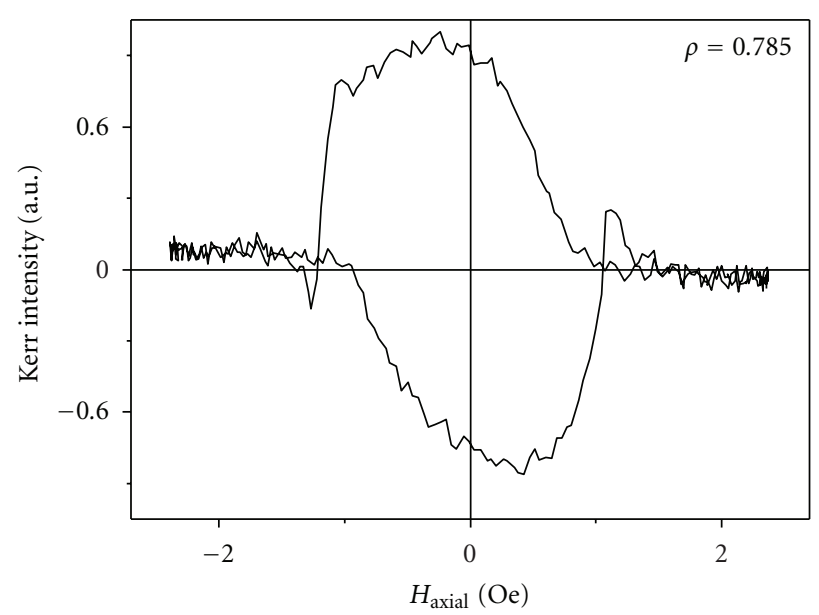

(a)

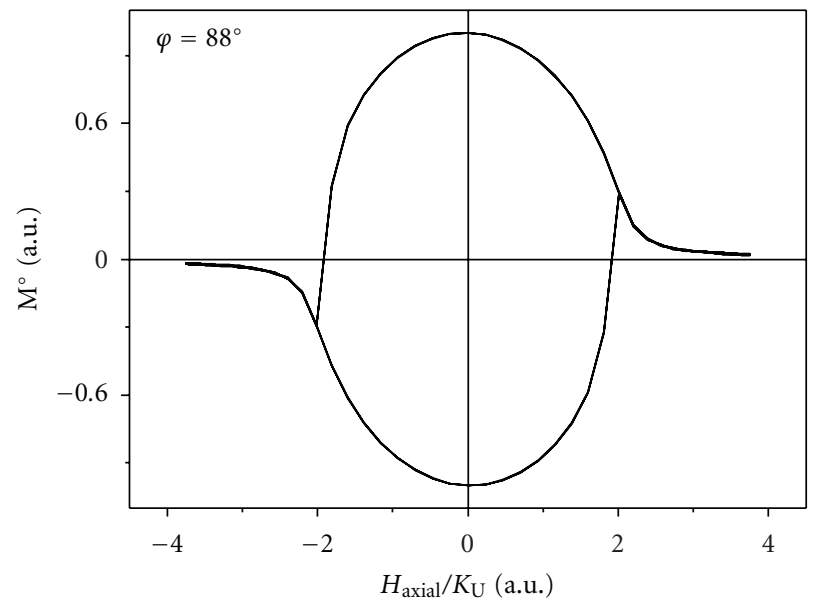

(c)

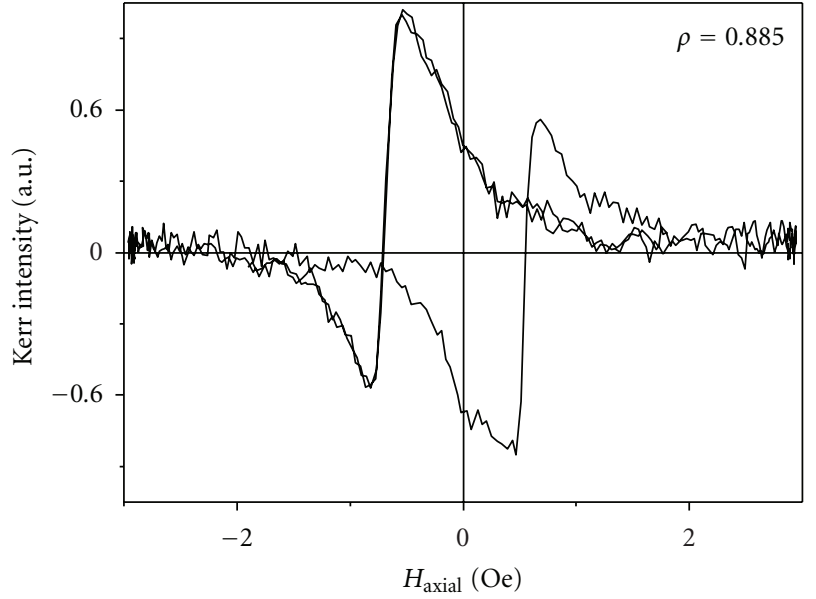

(b)

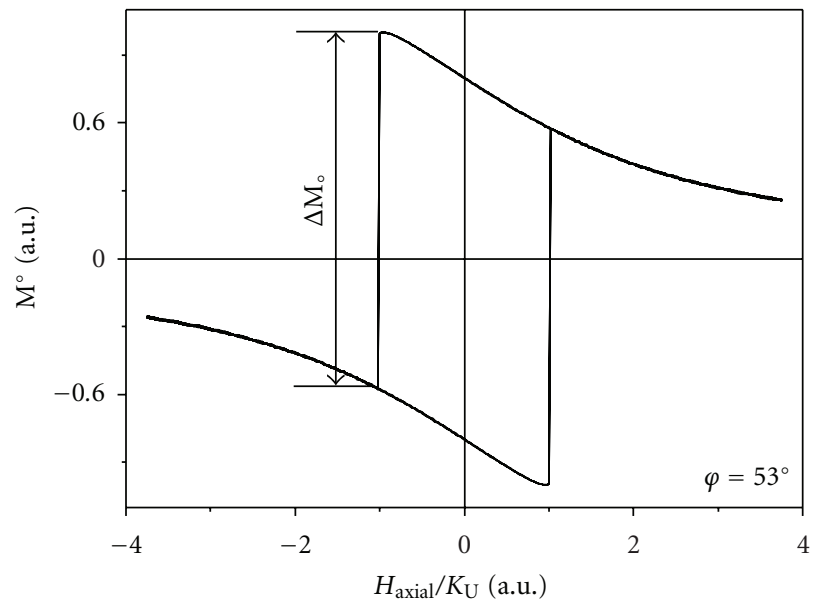

(d)

FIGURE 13: (a)-(b) experimental transverse Kerr effect dependencies on axial magnetic field for two wires with different thickness of glass covering. (c)-(d) calculated dependences of circular magnetization on normalized axial magnetic field for two angles of the anisotropy direction $\varphi$.

direction. For the angles close to $60^{\circ}$ the jump of the magnetization occurs at the moment when the direction of the magnetization is close to the circular magnetization. Because of that the value of the $\Delta M_{\text {CIRC }}$ is large for these angles. When the angle of helicality approximates to $0^{\circ}$, the inclination of the magnetization decreases. The Kerr effect experimental results and the results of the calculation have been compared taking into account the value and position of the jump of the circular magnetization $\Delta M_{\text {CIRC. }}$ This jump is related to the overcoming of the helical hard axis. The shape of the calculated hysteresis curves, and, in particular, the value of the jump $\Delta M_{\mathrm{CIRC}}$ is very sensitive to the value of the angle of the anisotropy. We can conclude that the angle of the helical anisotropy in the surface of the studied microwires is $88^{\circ} \pm 1^{\circ}$ for the wire with $\rho=0.785$ and $53^{\circ} \pm 1^{\circ}$ for the wire with $\rho=0.885$. The results of the comparison are presented in Figures 13(c) and 13(d) (transversal projection).

Based on the same calculation, we have analysed the bulk hysteresis curves (Figure 14), taking into account that these curves have the information about the axial magnetization reversal in the whole volume of the wire. Following the above-mentioned criteria, we have determined the angles of the helical anisotropy which could be attributed to the experimental volume hysteresis: $70^{\circ} \pm 1^{\circ}$ for the wire with $\rho=0.785$ and $45^{\circ} \pm 1^{\circ}$ for the wire with $\rho=0.885$.

As it is possible to see, the angles gotten from the analysis of the experimental results obtained by Kerr effect and the conventional method for the same microwires are different. The conventional curves present information about the magnetization reversal averaged through the wire volume, and the Kerr effect curves reflect the magnetization reversal in the thin surface layer of the wire. Based on the comparison of the obtained results, we can say that there is some distribution of the anisotropy angle in the wire, and that this angle increases in the approximation to the surface. The difference $\Delta$ between the averaged and the surface values of the angle is different for the two studied wires: 18 for the wire with $\rho=0.785$ and 8 for the wire with $\rho=0.885$. 


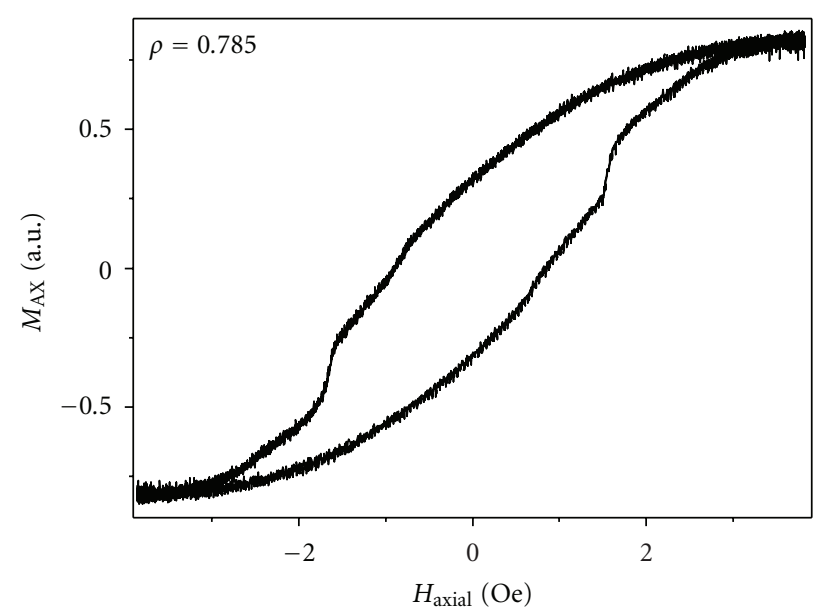

(a)

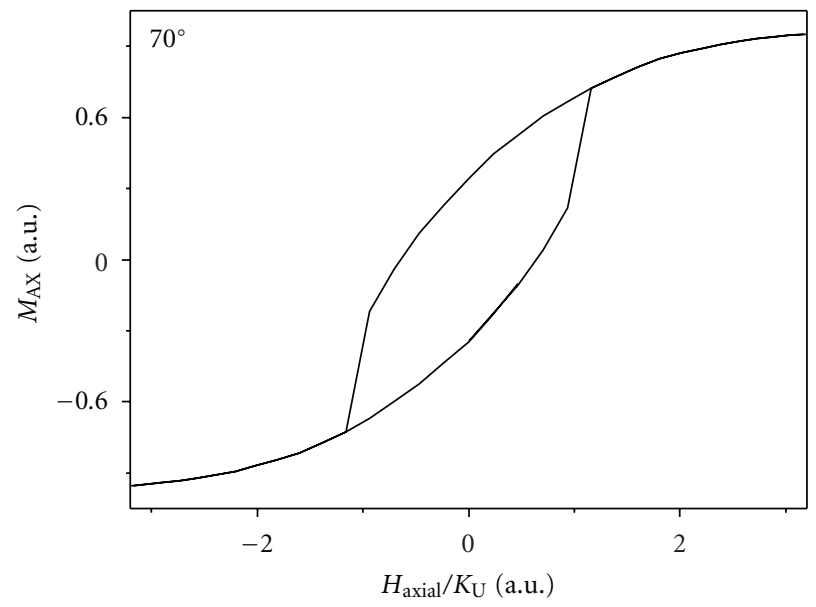

(c)

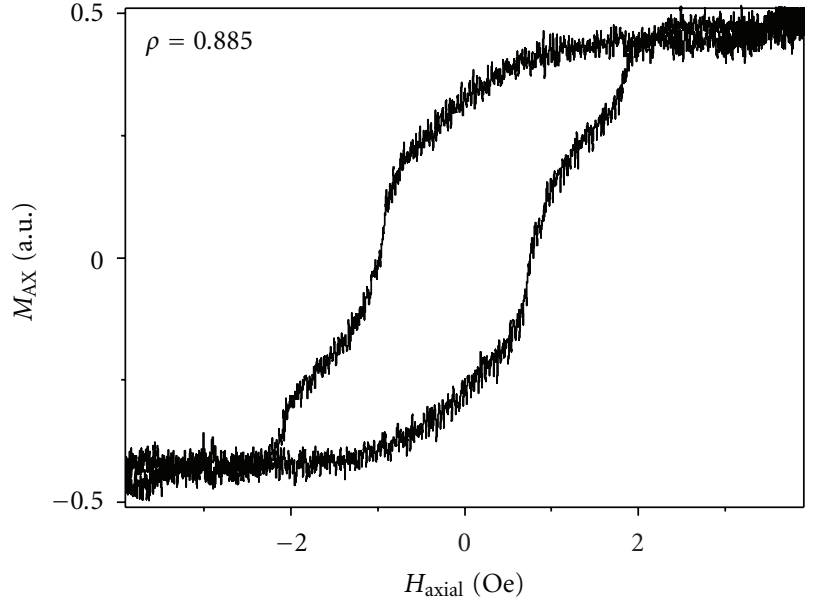

(b)

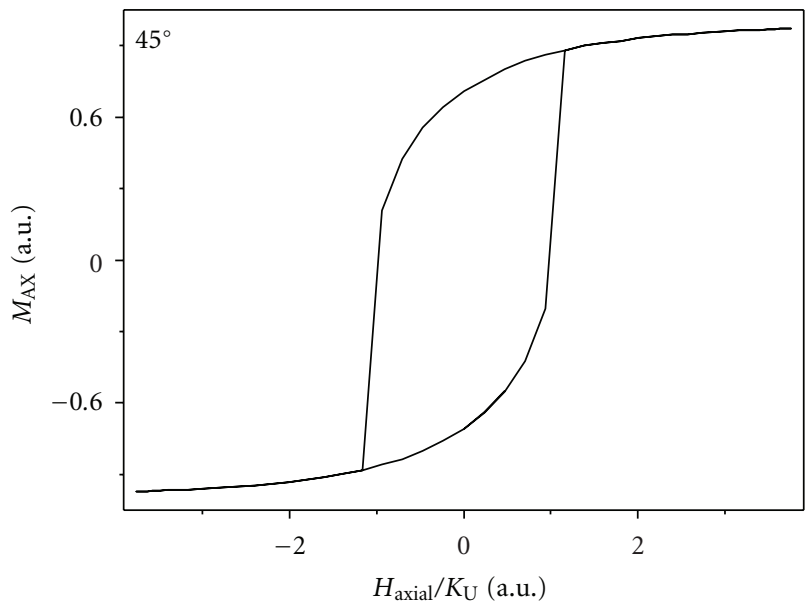

(d)

FIGURE 14: (a)-(b) experimental bulk dependencies on axial magnetic field for the two wires with different thickness of glass covering. (c)-(d) calculated dependencies of axial magnetization on normalized axial magnetic field for two angles of anisotropy direction $\varphi$.

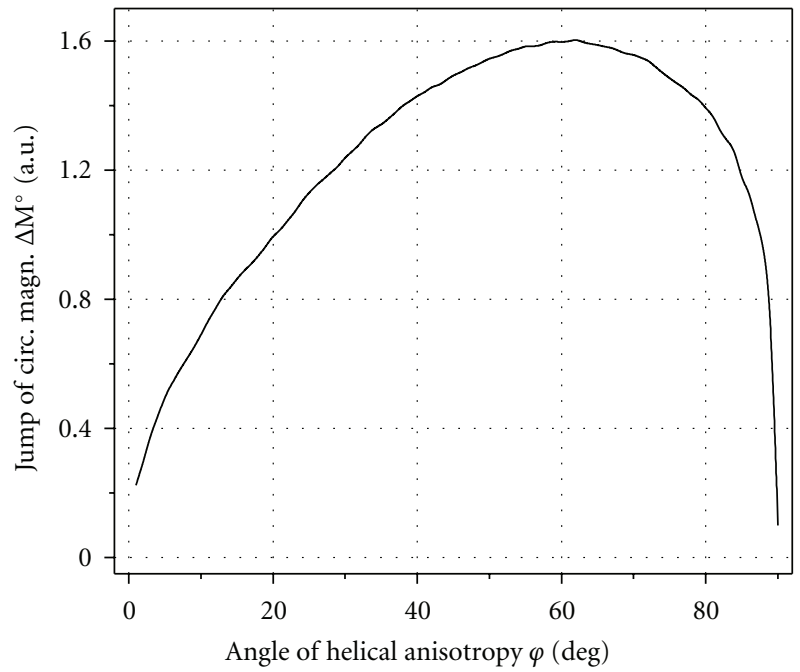

FIgure 15: Calculated dependence of the jump of the circular magnetization $\Delta M_{\mathrm{CIRC}}$ on the angle of helical anisotropy $\varphi$.
It could mean that the distribution of the anisotropy angle is more inhomogeneous for the wire with $\rho=0.785$. In turn, it is related to the different distribution of the internal stress inside the wire.

Frequency dependence of the coercive field has been studied for the wire with $\rho=0.885$ using the conventional and Kerr effect techniques. The results of the conventional experiments are presented in Figure 16. Frequency dependence of the coercive field $H_{C}$ has been analyzed using the calculations presented in [25]. It was shown that the result of the solution of the equation of domain wall motion in the following relation has been obtained [26]:

$$
H_{\mathrm{CD}}=H_{\mathrm{CO}}+\frac{4 f H_{0}\left(L+2 I_{\mathrm{S}} A\right)}{K},
$$

where $H_{\mathrm{CD}}$ is the dynamic switching field, $H_{\mathrm{CO}}$ is the static coercive field, $f$ is the frequency of the magnetic field, $H_{0}$ is the amplitude of the magnetic field, $L$ is the damping coefficient, $I_{\mathrm{S}}$ is the saturation magnetization, $K$ is the elastic 


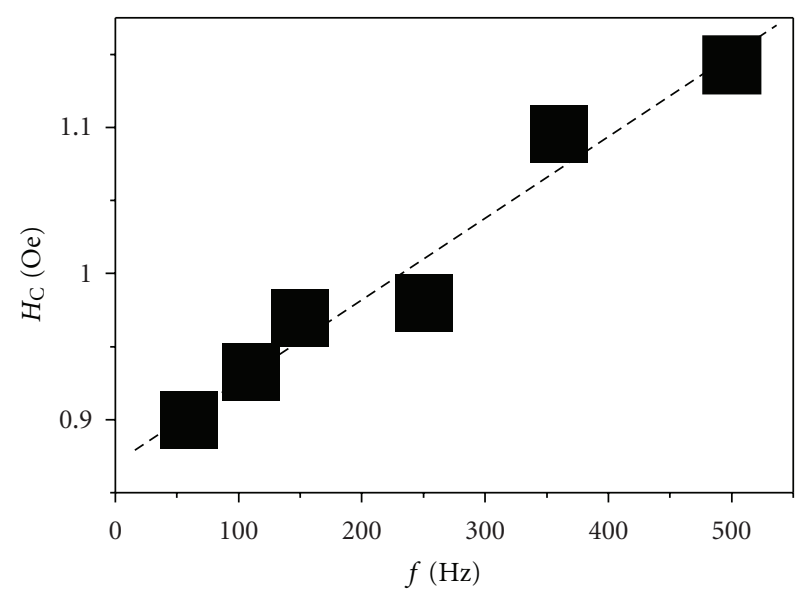

Figure 16: Dependence of the volume coercive field on the frequency of magnetic field for the wire with $\rho=0.885$.

coefficient, and $A$ is a proportionality constant. This linear dependence of the coercive field on frequency could be described in terms of domain nucleation mechanism. The nucleation process is associated with the overcoming of the energy barrier. Thermoactivation mechanism of the overcoming was satisfactorily employed for the explanation of the coercive field fluctuations observed in amorphous wires. Frequency of the applied field in this way affects the value of the coercive field. The experimental dependence of the coercive field on the frequency fits well the linear function that gives us possibility to conclude that the above mechanism of magnetization reversal could be applied to this experiment.

Kerr effect dependencies also changed when the frequency of the axial magnetic field increased. But the transformation of the surface curves differs from the transformation of the bulk curves. Figure 17(a) demonstrates the observed decrease of $H_{\mathrm{C}-\mathrm{MO}}$ and the increase of $I_{\mathrm{MAX}}\left(H_{\mathrm{C}-\mathrm{MO}}\right.$ should be considered as the field at which the drastic change of the circular magnetization starts, $I_{\mathrm{MAX}}$ is the maximal intensity of the Kerr signal during the magnetization reversal). Taking into account that the Kerr intensity is proportional to the transverse magnetization in the surface area of the wire, $I_{\mathrm{MAX}} \sim M_{\mathrm{MAX}}$, where $M_{\mathrm{MAX}}$ is the maximal value of the transverse (circular) magnetization.

Analyzing the results obtained in the Kerr effect experiments, we consider that the magnetization reversal process in the surface area of the wire occurs as the formation of a circular domain in the outer shell. The coercive field $H_{\mathrm{C}-\mathrm{MO}}$ is, respectively, determined by the circular domain nucleation. The expression for $H_{\mathrm{C}-\mathrm{MO}}$ can be presented as [26]

$$
H_{\mathrm{C}-\mathrm{MO}}=\frac{\alpha}{M_{\mathrm{MAX}}}-N M_{\mathrm{MAX}}
$$

where the first term is related to losses of energy at nucleation process and the second term to the demagnetizing field of the nucleus ( $N$ is the demagnetizing factor). We consider, within numerical factor, that $\alpha=\sigma / v^{1 / 3}$, where $\sigma$ is energy of domain wall, $v$ is critical volume of the nucleus.

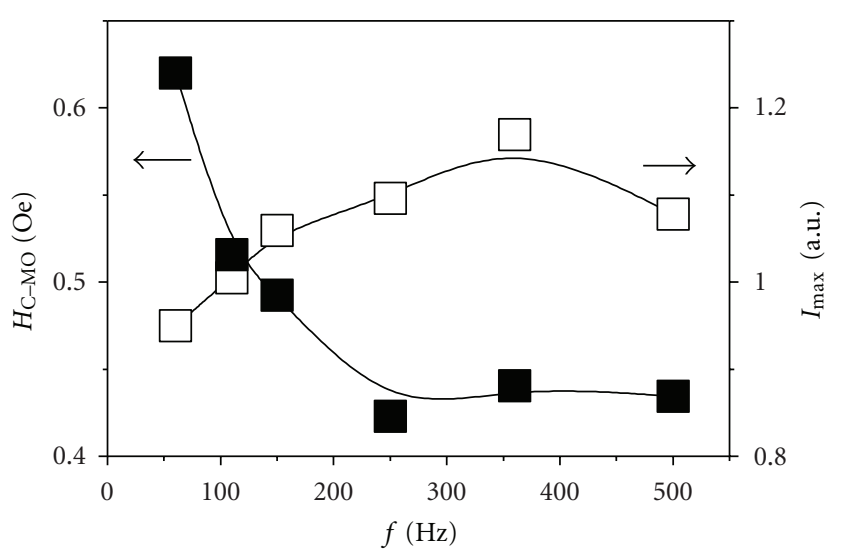

(a)

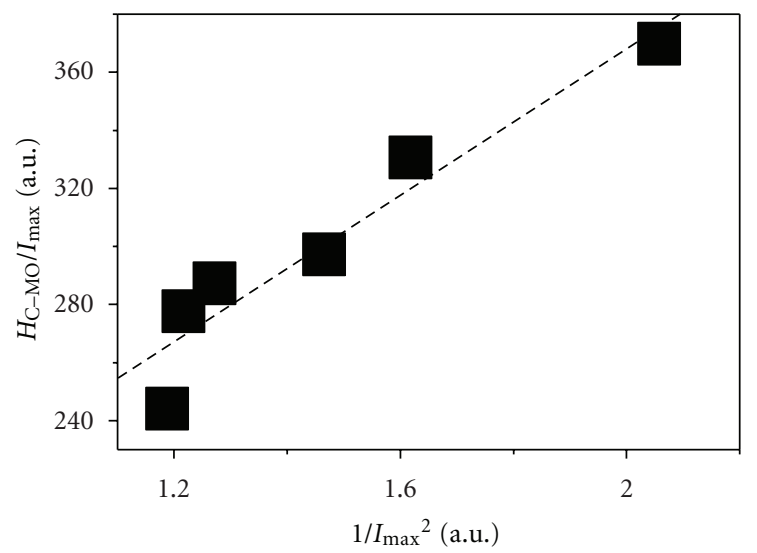

(b)

Figure 17: (a) $H_{\mathrm{C}-\mathrm{MO}}$ and $I_{\mathrm{MAX}}$ dependencies on frequency of magnetic field for $\rho=0.885$; (b) $H_{\mathrm{C}-\mathrm{MO}} / M_{\mathrm{MAX}}$ dependence on $1 / M_{\mathrm{MAX}^{2}}$.

To verify the above-mentioned assumption regarding the mechanism of the magnetization reversal in the outer shell of the microwire, the $H_{\mathrm{C}-\mathrm{MO}} / I_{\mathrm{MAX}}$ dependence on $1 / I_{\mathrm{MAX}}{ }^{2}$ has been plotted (Figure 17(b)). Taking into account that $I_{\mathrm{MAX}} \sim$ $M_{\mathrm{MAX}}$, this dependence could be considered as the analogy of $H_{\mathrm{C}-\mathrm{MO}} / \mathrm{M}_{\mathrm{MAX}}\left(1 / \mathrm{M}_{\mathrm{MAX}}{ }\right)$ dependence. Good fitting of the experimental points by the linear function demonstrates the strong relation between the $H_{\mathrm{C}-\mathrm{MO}}$ and the $M_{\mathrm{CIRC}}$.

The strong difference of the frequency dependence of the $H_{\mathrm{C}}$ and $H_{\mathrm{C}-\mathrm{MO}}$ means that the magnetization reversal happens independently enough in the surface and in the volume of the wire. $H_{\mathrm{C}-\mathrm{MO}}$ is smaller than $H_{\mathrm{C}}$. Therefore, the nucleation starts in the surface in the moment when the circular projection of the magnetization reaches the sufficient value. This value of this circular projection is the key parameter which changes with the magnetic field frequency. We suppose that one of the possible reasons of this change is the following one. The increase of the frequency means the growth of the velocity of the increase of the magnetic field $d H / d t$. Therefore, the magnetic field increases more quickly for the higher frequency. The quicker increase of magnetic field causes the quicker increase of the circular magnetization. Therefore, for the higher frequency 


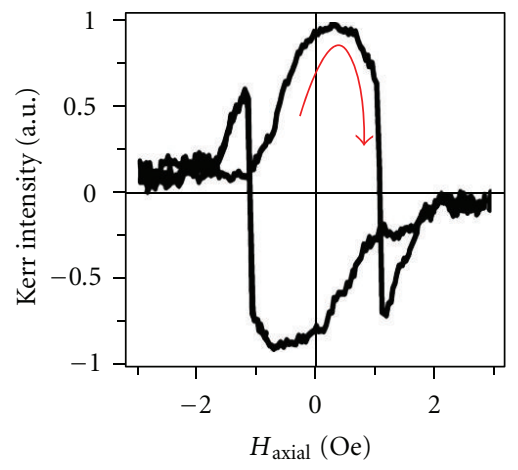

(a)

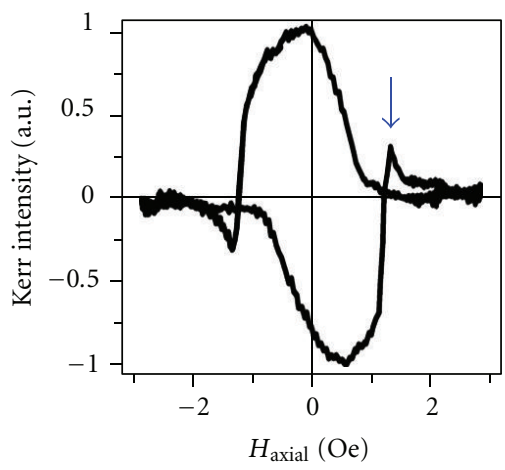

(c)
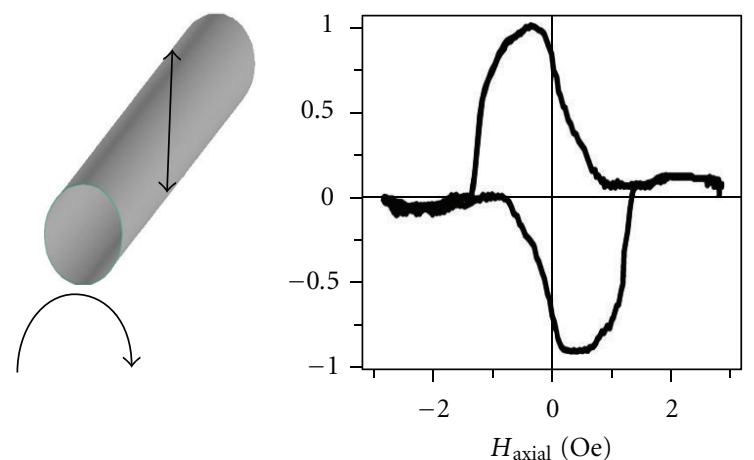

(b)
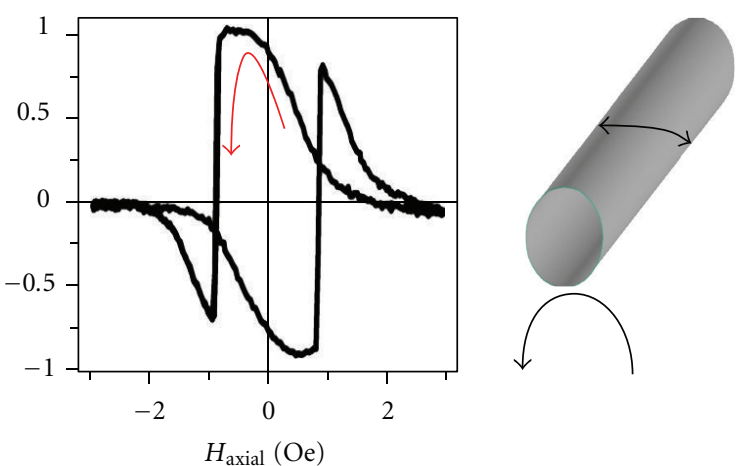

Figure 18: Transverse Kerr effect dependence on the magnetic field applied along the microwire axis in the presence of torsion stress. (a) $\tau=-22 \pi \mathrm{rad} \mathrm{m}^{-1}$, (b) $\tau=-2.2 \pi \mathrm{rad} \mathrm{m}^{-1}$, (c) $\tau=0$, and (d) $\tau=8.9 \pi \mathrm{rad} \mathrm{m}^{-1}$. Insets: schematically pictures of the inclination of the axis of helical anisotropy induced by the torsion stress.

the magnetization could reach the larger value in the moment when the domain nucleus becomes the stable one, that is, in the moment close to the nucleation. Finally, the relation of the $d H / d t$ and the probability of the nucleation are realized in the decrease of the $H_{\mathrm{C}-\mathrm{MO}}$ with frequency.

We consider that the surface magnetization reversal is determined mainly by the nucleation mechanism. The sharpness of the $\Delta M_{\text {CIRC }}$ jump (Figure 13(b)) and our early Kerr microscopy study of the magnetization reversal in amorphous wires [27] permit us to make such conclusion.

Equation (7) is used in general for the temperature dependence of the coercive field. Earlier we have used it for the frequency dependence of the coercive field [28], and we believe that this application is reasonable taking into account the similar character of the influence of the temperature and frequency on the probability of the domain nucleation.

For the determination of the angle of helical anisotropy, we applied the calculation which did not include the domain wall motion, while for the frequency experiments we use the equation which takes into account the nucleation and domain wall motion. For the studied case, the shape of the hysteresis curve and, particularly, the value and the position of the $\Delta M_{\text {CIRC }}$ jump, which is the value we used in the analysis, are determined mainly by the direction of the helical anisotropy and to a lesser degree, by the details of the domain wall motion. But for the dependencies of the coercive field on the frequency, the features of the magnetization reversal become important, Such as known [25], the change of the frequency of the external magnet field influences strongly on the mechanism of the magnetic reversal in the wires.

\section{Torsion Stress-Induced Transformation of Surface Magnetic Structure}

The process of surface magnetization reversal in the presence of torsion stress of different amplitudes and directions has been studied in microwire of nominal composition of $\mathrm{Co}_{69.5} \mathrm{Fe}_{3.9} \mathrm{Ni}_{1} \mathrm{~B}_{11.8} \mathrm{Si}_{10.8} \mathrm{Mo}_{2}(\rho=0.79)$ (Figure 18) [29].

The magnetization reversal in the surface area of the wires has been studied by transverse magneto-optical Kerr effect method in the presence of axial magnetic field. The torsion stress up to $40 \pi \mathrm{rad} \mathrm{m}^{-1}$ has been applied during the experiments.

The surface magnetization reversal consists of two steps: the magnetization rotation from axial to circular direction in the outer shell following by the magnetization jump between two states with opposite directions of surface circular magnetization. The applied torsion stress induces strong transformation of the surface hysteresis loops. The most essential feature of this transformation is a stress-induced change 


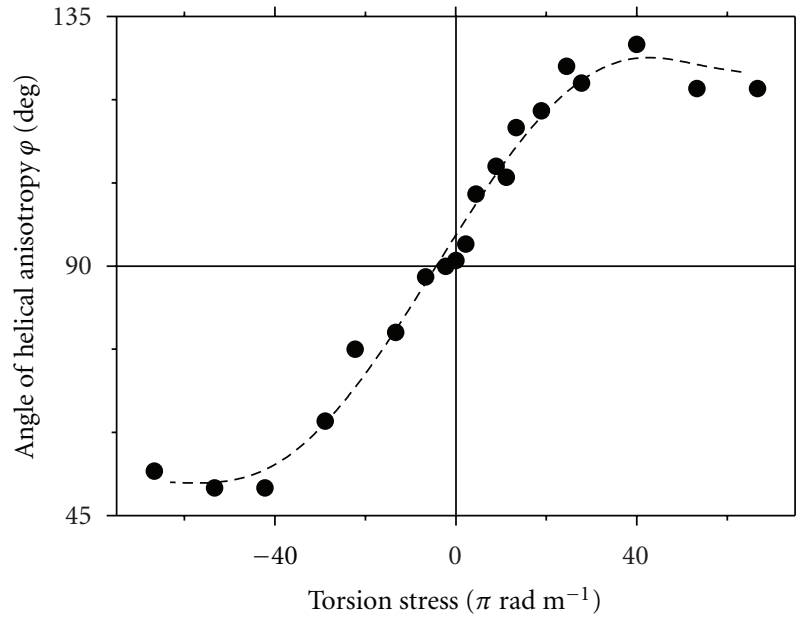

Figure 19: Dependence of angle of helical anisotropy on applied torsion stress.

in the value and direction (sign) of the jump of Kerr intensity $\Delta I$ (the circular magnetization $\Delta M_{\text {CIRC }}$ ).

It was experimentally confirmed, as predicted, that the torsion stress-induced inclination does not exceed the value of $45^{\circ}$. This inclination along with the growth of the value of the helical anisotropy is the key phenomenon determining the main mechanism of the surface magnetization reversal. As a helical anisotropy increases, this mechanism greatly changes.

The value of jump of the circular magnetization $\Delta M_{\text {CIRC }}$ for different values of torsion stress was obtained experimentally, and also, by calculating the dependence of the circular magnetization jump $\Delta M_{\mathrm{CIRC}}$ on the angle of helical anisotropy. Based on numerical analysis of the obtained experimental results, the dependence of helical anisotropy angle on the torsion stress has been obtained for the first time in amorphous wires (Figure 19).

\section{Surface Magnetic Domains Nucleation and Transformation}

The evolution of the domain structure during the magnetization reversal processes was studied in microwires with nominal composition $\mathrm{Co}_{67} \mathrm{Fe}_{3.85} \mathrm{Ni}_{1.45} \mathrm{~B}_{11.5} \mathrm{Si}_{14.5} \mathrm{Mo}_{1.7}$ with geometric ratio $\rho=0.79$ in the presence of circular magnetic field [30].

The circular magnetic domains could be observed because of the out-of-plane components of the surface magnetization that transforms to black-white contrast when the polarized light reflects from the cylindrical-shape surface of the microwire. The observations of circular magnetic domain images structures were performed by optical polarizing microscope using polar magneto-optical Kerr effect geometry. In this configuration, we can observe the magnetic areas with out-of-plane component magnetization concerning an optical axis of objective and identify domains with the magnetization of opposite directions. "Leica" microscope was (a)

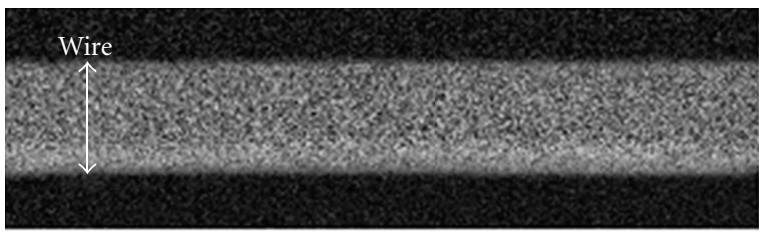

(b)

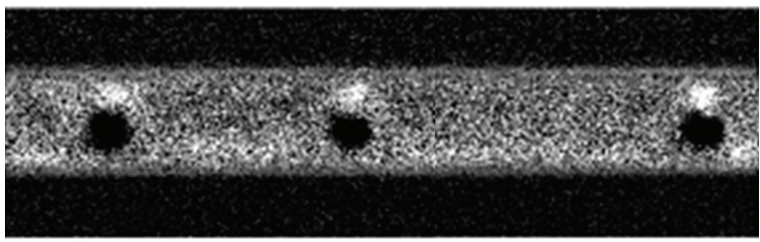

(c)

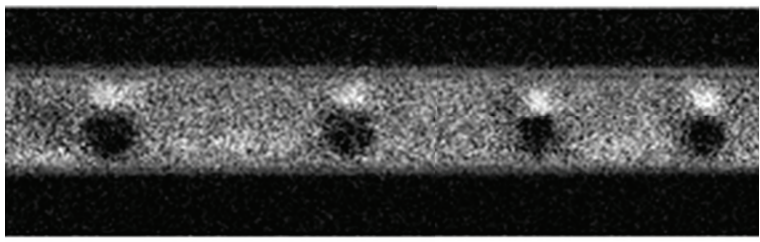

(d)
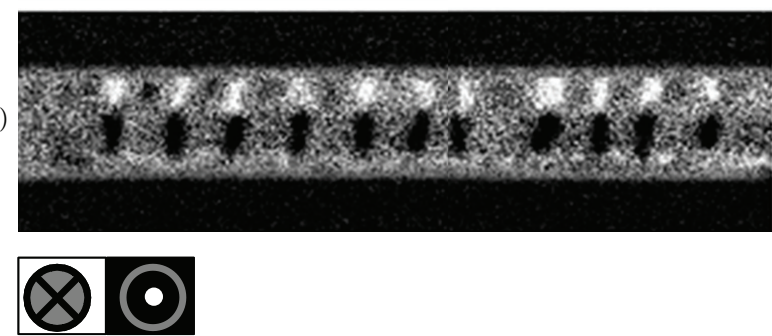

FIGURE 20: The images of magnetic domain structure registered in different values of circular magnetic field: (a) 0, (b) $0.3 \mathrm{Oe}$, (c) $0.36 \mathrm{Oe}$, and (d) 0.45 Oe. Image size is $170 \times 50 \mu \mathrm{m}^{2}$.

equipped with halogen lamp and high-sensitivity "Hamamatsu" digital charge-coupled device (CCD) camera. The following procedure was used for the registration of the surface magnetic domain image: the microwire was saturated in circular magnetic field, then the field was reduced to zero, and the reference image was recorded. In the next step, images in real time during the increase of the magnetic field were registered. After image processing, each image was a result of difference between two images: domain structure at various times and the reference image.

The results of the polar MOKE microscopy experiments are presented in Figure 20. The magnetization reversal starts from a single-domain state (Figure 20(a)). We noticed that the magnetization reversal could be originated at any part of the microwire. At the first stage of the magnetization reversal, the nucleation and fast domain wall motion cause the formation of relatively small circular domains Figure 20(b). Increasing the circular magnetic field leads to the increase in the number of domains with practically equal width Figure 20(c). At the next stage, a strong rearrangement of the domain structure takes place: the number of domains increases sharply Figure 7(d). The magnetization reversal finishes with the formation of a reversed single-domain state.

The magnetization reversal occurs mainly by domain nucleation that is determined basically by the local nucleation sites distribution. The irregular rearrangement of 

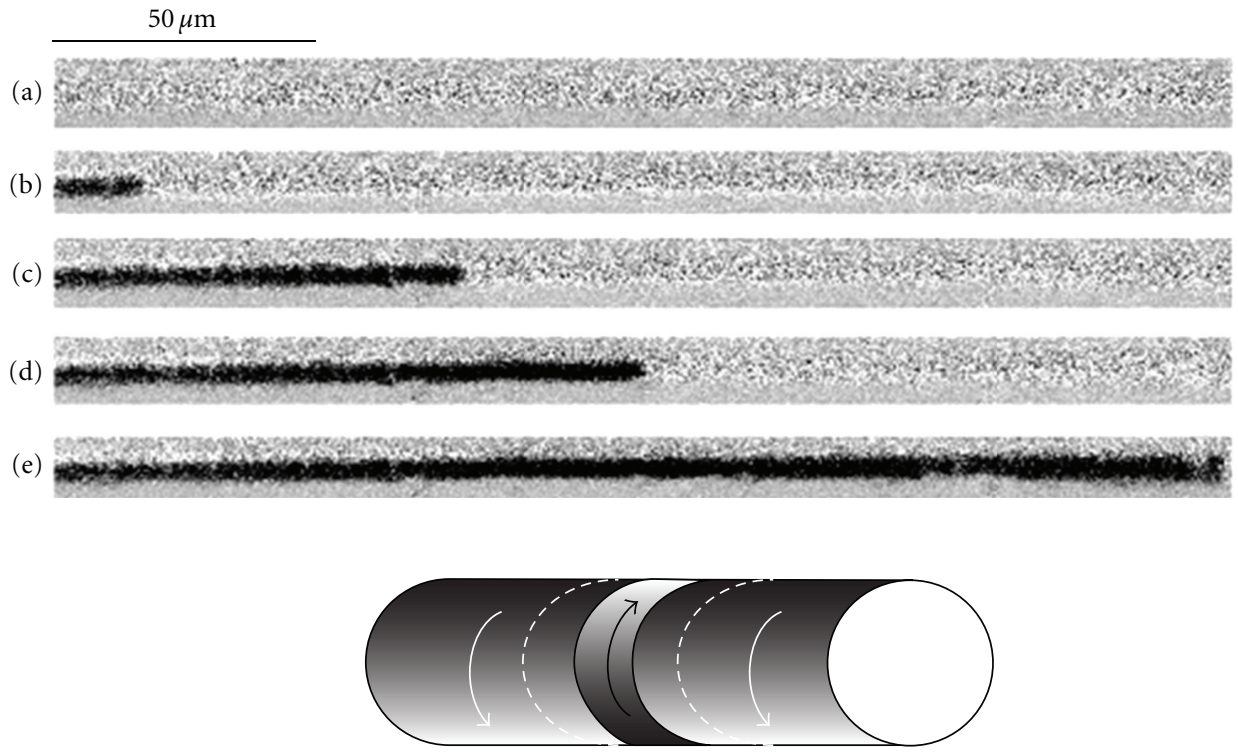

FIGURE 21: Photographs of circular domain formation and domain walls propagation in the presence of circular field of constant amplitude and increasing time duration, $I=0.38 \mathrm{~mA}, H_{\mathrm{AX}}=0.27$ Oe. (a) $t=0$, (b) $t=310 \mathrm{msec}$, (c) $t=372 \mathrm{msec}$, (d) $t=434 \mathrm{msec}$, and (e) $t=496 \mathrm{msec}$.

the circular domain structure takes place during the magnetization reversal that was not observed earlier in amorphous magnetic microwires. The circular magnetic domain nucleation in amorphous microwires could be described successfully by the thermoactivated mechanism.

\section{Observation of Circular Giant Barkhausen Jumps}

First, surface magnetization reversal has been studied using MOKE magnetometer as a function of circular magnetic field in the presence of axial DC bias field in microwire with nominal composition $\mathrm{Co}_{67} \mathrm{Fe}_{3.85} \mathrm{Ni}_{1.45} \mathrm{~B}_{11.5} \mathrm{Si}_{14.5} \mathrm{Mo}_{1.7}$ $(\rho=0.79)$ [31]. The jumps of the circular magnetization, associated with Barkhausen jumps, have been observed. These jumps are related to the mentioned effect of circular magnetic bistability. It was found that the application of the axial magnetic field transforms the hysteresis curve in such a way, that the jump of the circular magnetization becomes sharper. Observed change of the hysteresis loop is related to the transformation of the helical magnetic structure induced by the axial magnetic field. In the presence of the axial field of some special value, the jump occurs between two circularly magnetized states.

We have two processes with different velocities of the magnetization reversal. We assume that this difference in velocity reflects the difference in the process of domain nucleation and domain walls propagation. To verify this supposition and to elucidate the details of the Barkhausen jumps, we have performed the MOKE microscope experiments (Figure 21).

Observation of the circular magnetic domain structures in microwires was performed by an optical polarizing microscope to detect the longitudinal MOKE. A Carl Zeiss Jenapol microscope was equipped with Xe-lamp and a Cool SNAP (Princeton Instruments) high-sensitivity camera having a $1300 \times 1030$ pixels, cooled CCD element. The magnetic contrast of the domain structure was improved by image processing software based on LABVIEW.

Figure 21 shows the surface magnetization reversal for the case of $H_{\mathrm{AX}} \neq 0$. The successive increase of the time duration of the constant circular magnetic field induces the nucleation of solitary circular domain following by the propagation of the domain wall moving for a long distance along the microwire. This scenario could be considered as classical realization of the giant Barkhausen jump.

Using the axial magnetic field as a unique external parameter, we have achieved that the surface magnetization reversal realizes in the form of sharp giant Barkhausen jump magnetic microwires. We have demonstrated for the first time that this jump is characterized by the quick motion of the solitary domain wall which overpasses long distances of about hundreds of micrometers. We have shown that the extensive motion of the solitary domain wall really exists in magnetic wires, and that it is the determinative constituent of the surface giant Barkhausen jump.

\section{Conclusions}

The high GMI ratio can be observed in thin glass-coated ferromagnetic microwires with adequate chemical composition and geometry. Changing both parameters, we are able to tailor the GMI ration in Co-rich microwires. It was found that the induced transverse magnetic anisotropy allows us to predict the existence of the magneto-impedance effect. Application of the MOKE method allowed to correlate the axial magnetic field dependences of GMI ratio and MOKE hysteresis loops: a maximum of GMI ratio occurs 
approximately at the same field as the sharp change of the circular magnetization on the surface loop.

Taking into account the essential role of the helical magnetic anisotropy in the GMI effect, the magnetization reversal has been studied in the Co-rich microwires with helical magnetic structure. The value of the angle of the helical anisotropy in the surface and in the volume of the wires was determined. Also, it was found that the increase of the frequency of the external axial magnetic field induces the change of the coercive properties in the volume and in the surface of the microwire: the coercive field of the surface magnetic structure decreases with the frequency of the magnetic field when the volume coercive field increases. The surface coercive field is mainly determined by the value of the magnetization in the outer shell of the wire.

The surface and bulk measurements of Co-rich microwires with different chemical composition (especially, the different $\mathrm{Mn}$ content) demonstrate the variety of the shapes of magnetization reversal loops, that can be attributed to the change of sign of the magnetostriction. The rectangular shape of the surface hysteresis loop in circular magnetic field of the microwire with $x=0.07$ can be interpreted by considering that the magnetization process takes place by large Barkhausen jump of circular domain structure, while the rectangular shape of the hysteresis loop in axial magnetic field of the microwire with $x=0.11$ could be attributed to the large Barkhausen jump within the axial domain structure.

The circular bistability and related circular giant Barkhausen jump are the basic effects which determine the character of the surface magnetization reversal. The giant Barkhausen jump is followed by the formation of the circular domain structure at the surface of the microwire. At the special conditions the long-distance motion of the solitary circular domain wall is observed as a classical realization of the giant Barkhausen jump.

The strong transformation of the surface domain structure takes place in the presence of tensile and torsion external stresses. The direct relation between the angle of helical anisotropy and the value of the torsion stress has been established. The limit values of the angle of the helical anisotropy have been experimentally determined.

\section{References}

[1] L. V. Panina and K. Mohri, "Magneto-impedance effect in amorphous wires," Applied Physics Letters, vol. 65, no. 9, pp. 1189-1191, 1994.

[2] V. Zhukova, A. Chizhik, A. Zhukov, A. Torcunov, V. Larin, and J. Gonzalez, "Optimization of giant magnetoimpedance in Corich amorphous microwires," IEEE Transactions on Magnetics, vol. 38, no. 5 I, pp. 3090-3092, 2002.

[3] A. Zhukov, V. Zhukova, J. M. Blanco, and J. Gonzalez, "Recent research on magnetic properties of glass-coated microwires," Journal of Magnetism and Magnetic Materials, vol. 294, no. 2, pp. 182-192, 2005.

[4] A. Zhukov, J. González, M. Vázquez, V. Larin, and A. Torcunov, "Nanocrystalline and amorphous magnetic microwires," in Encyclopedia of Nanoscience and Nanotechnology, H. S.
Nalwa, Ed., vol. 23, chapter 62, pp. 1-22, American Scientific Publishers, 2004.

[5] R. Hasegawa, "Nonmagnetostrictive glassy Co-Fe-Ni-Mo-B-Si alloys," Journal of Applied Physics, vol. 53, no. 11, pp. 78197821, 1982.

[6] A. Zhukov, J. M. Blanco, J. González, M. J. G. Prieto, E. Pina, and M. Vázquez, "Induced magnetic anisotropy in Co-Mn-SiB amorphous microwires," Journal of Applied Physics, vol. 87, no. 3, pp. 1402-1409, 2000.

[7] A. F. Cobeño, A. Zhukov, A. R. De Arellano-López et al., "Physical properties of nearly zero magnetostriction Co-rich glasscoated amorphous microwires," Journal of Materials Research, vol. 14, no. 9, pp. 3775-3783, 1999.

[8] A. Chizhik, J. Gonzalez, A. Zhukov, and J. M. Blanco, "Magnetization reversal of Co-rich wires in circular magnetic field," Journal of Applied Physics, vol. 91, no. 1, pp. 537-539, 2002.

[9] M. Knobela, C. Gómez-Polo, and M. Vázquez, "Evaluation of the linear magnetostriction in amorphous wires using the giant magneto-impedance effect," Journal of Magnetism and Magnetic Materials, vol. 160, pp. 243-244, 1996.

[10] J. Velázquez, M. Vázquez, and A. P. Zhukov, "Magnetoelastic anisotropy distribution in glass-coated microwires," Journal of Materials Research, vol. 11, no. 10, pp. 2499-2505, 1996.

[11] A. F. Cobeo, A. Zhukov, J. M. Blanco, and J. Gonzalez, "Giant magneto-impedance effect in $\mathrm{CoMnSiB}$ amorphous microwires," Journal of Magnetism and Magnetic Materials, vol. 234, no. 3, pp. L359-L365, 2001.

[12] N. A. Usov, A. S. Antonov, and A. N. Lagar'kov, "Theory of giant magneto-impedance effect in amorphous wires with different types of magnetic anisotropy," Journal of Magnetism and Magnetic Materials, vol. 185, no. 2, pp. 159-173, 1998.

[13] J. Velázquez, M. Vázquez, and A. P. Zhukov, "Magnetoelastic anisotropy distribution in glass-coated microwires," Journal of Materials Research, vol. 11, no. 10, pp. 2499-2505, 1996.

[14] J. González, K. Kułakowski, P. Aragoneses, J. M. Blanco, and E. Irurieta, "Stress dependence of bistability in a zero-magnetostrictive amorphous wire," Journal of Materials Science, vol. 30, no. 20, pp. 5173-5177, 1995.

[15] J. González, A. Chizhik, A. Zhukov, and J. M. Blanco, "Surface magnetic behavior of nearly zero magnetostrictive Co-rich amorphous microwires," Journal of Magnetism and Magnetic Materials, vol. 258-259, pp. 177-182, 2003.

[16] F. B. Humphrey, K. Mohri, J. Yamasaki, H. Kawamura, R. Malmhäll, and I. Ogasawara, "Large barkhausen discontinuities in co-based amorphous wires with negative magnetostriction," in Magnetic Properties of Amorphous Metals, A. Hernando, V. Madurga, M. C. Sánchez-Trujillo, and M. Vázquez, Eds., p. 110, North-Holland, Amsterdam, The Netherlands, 1987.

[17] A. Chizhik, C. Garcia, J. Gonzalez, A. Zhukov, and J. M. Blanco, "Study of surface magnetic properties in Co-rich amorphous microwires," Journal of Magnetism and Magnetic Materials, vol. 300, no. 1, pp. e93-e97, 2006.

[18] B. K. Ponomarev and A. P. Zhukov, "Fluctuations of start field exhibited by amorphous alloy," Soviet Physics, Solid State, vol. 26, p. 2974, 1984.

[19] K. Mohri and S. Takeuchi, "Sensitive bistable magnetic sensors using twisted amorphous magnetostrictive ribbons due to Matteucci effect," Journal of Applied Physics, vol. 53, no. 11, pp. 8386-8388, 1982.

[20] L. Kraus, S. N. Kane, M. Vázquez et al., "Tensor components of the magnetization in a twisted Fe-rich amorphous wire," Journal of Applied Physics, vol. 75, no. 10, pp. 6952-6954, 1994. 
[21] J. González, N. Murillo, V. Larin, J. M. Barandiarán, M. Vázquez, and A. Hernando, "Magnetoelastic behavior of glasscovered amorphous ferromagnetic microwire," IEEE Transactions on Magnetics, vol. 33, no. 3, pp. 2362-2365, 1997.

[22] V. Zhukova, N. A. Usov, A. Zhukov, and J. Gonzalez, "Length effect in a Co-rich amorphous wire," Physical Review B, vol. 65, no. 13, Article ID 134407, 7 pages, 2002.

[23] A. Chizhik, J. M. Blanco, A. Zhukov et al., "Surface and bulk magnetic hysteresis loops of Co-rich glass covered microwires," IEEE Transactions on Magnetics, vol. 42, no. 12, pp. 3889-3892, 2006.

[24] M. Vázquez and A. Hernando, "A soft magnetic wire for sensor applications," Journal of Physics D, vol. 29, no. 4, pp. 939-949, 1996.

[25] A. Zhukov, M. Vázquez, J. Velázquez, C. García, R. Valenzuela, and B. Ponomarev, "Frequency dependence of coercivity in rapidly quenched amorphous materials," Materials Science and Engineering A, vol. 226-228, pp. 753-756, 1997.

[26] H. Kronmuller, "Micromagnetic background of hard magnetic materials," in Supermagnets, Hard Magnetic Materials, G. J. Long and F. Grandjean, Eds., p. 461, Kluwer Academic, Dodrecht, The Netherlands, 1991.

[27] A. Chizhik, J. Gonzalez, J. Yamasaki, A. Zhukov, and J. M. Blanco, "Vortex-type domain structure in Co-rich amorphous wires," Journal of Applied Physics, vol. 95, no. 5, pp. 2933-2935, 2004.

[28] A. Chizhik, A. Zhukov, J. Gonzalez, and J. M. Blanco, "Magneto-optical investigation of high-frequency electric current influence on surface magnetization reversal in Co-rich amorphous microwires," Journal of Applied Physics, vol. 97, no. 7, Article ID 073912, pp. 1-6, 2005.

[29] A. Chizhik, J. Gonzalez, P. Gawronski, K. Kulakowski, A. Zhukov, and J. M. Blanco, "Torsion and tension stress induced transformation of surface magnetic structure in Co-rich amorphous microwires," Journal of Non-Crystalline Solids, vol. 353, no. 8-10, pp. 935-937, 2007.

[30] A. Chizhik, A. Zhukov, A. Stupakiewicz, A. Maziewski, J. M. Blanco, and J. Gonzalez, "Kerr microscopy study of magnetic domain structure changes in amorphous microwires," IEEE Transactions on Magnetics, vol. 45, no. 10, Article ID 5257388, pp. 4279-4281, 2009.

[31] A. Chizhik, A. Stupakiewicz, A. Maziewski, A. Zhukov, J. Gonzalez, and J. M. Blanco, "Direct observation of giant Barkhausen jumps in magnetic microwires," Applied Physics Letters, vol. 97, no. 1, Article ID 012502, 2010. 

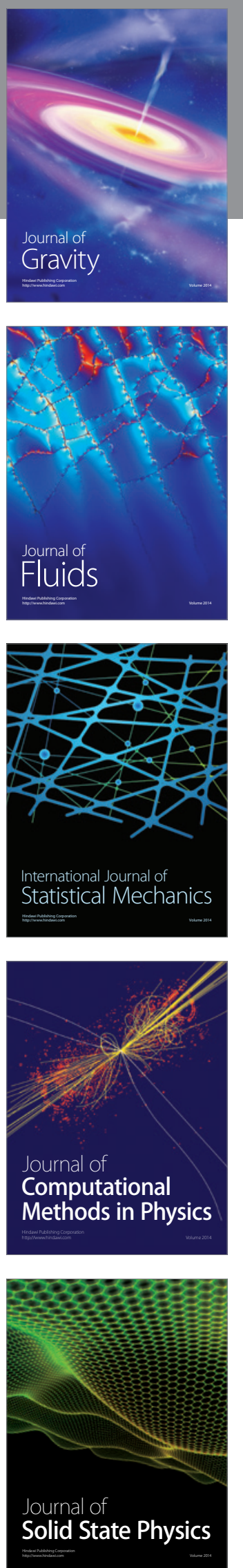

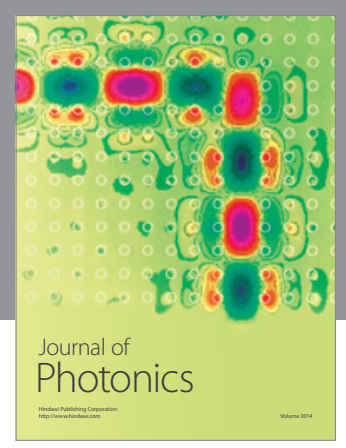

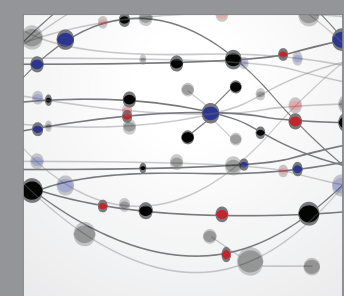

The Scientific World Journal
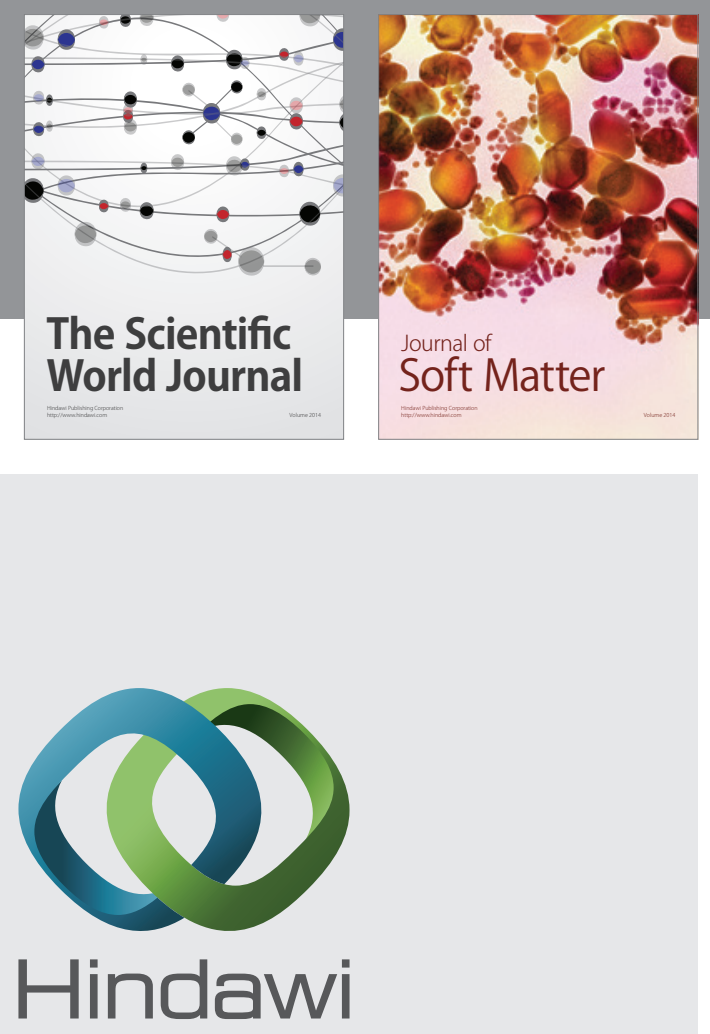

Submit your manuscripts at

http://www.hindawi.com
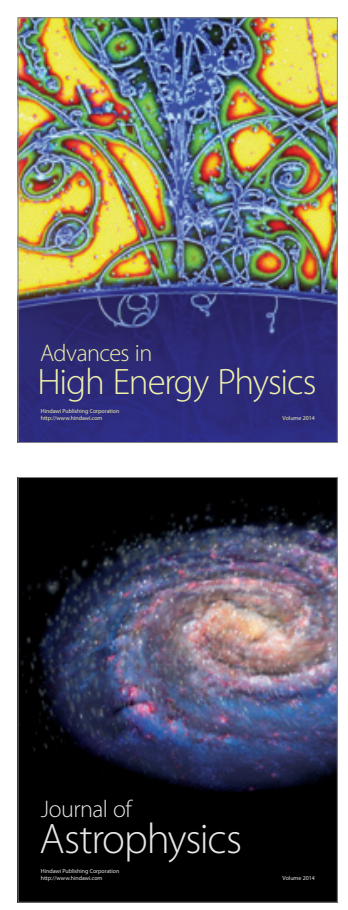
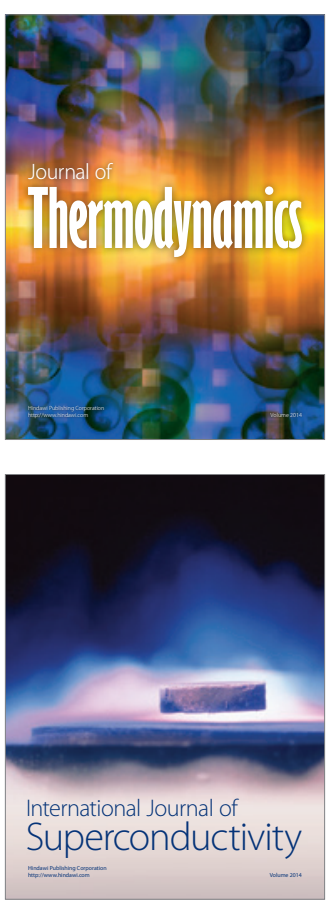
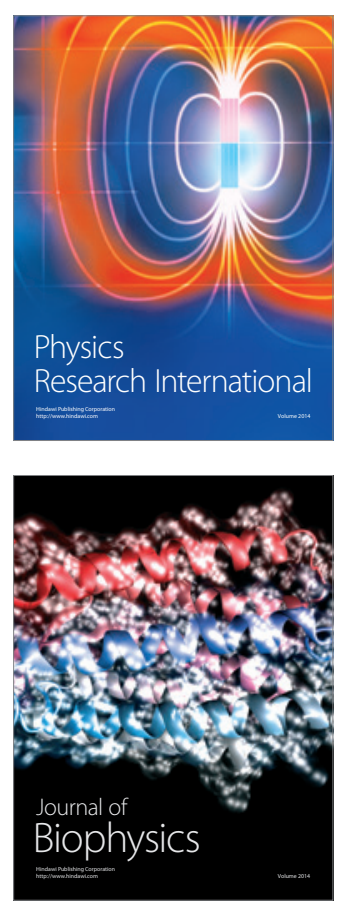
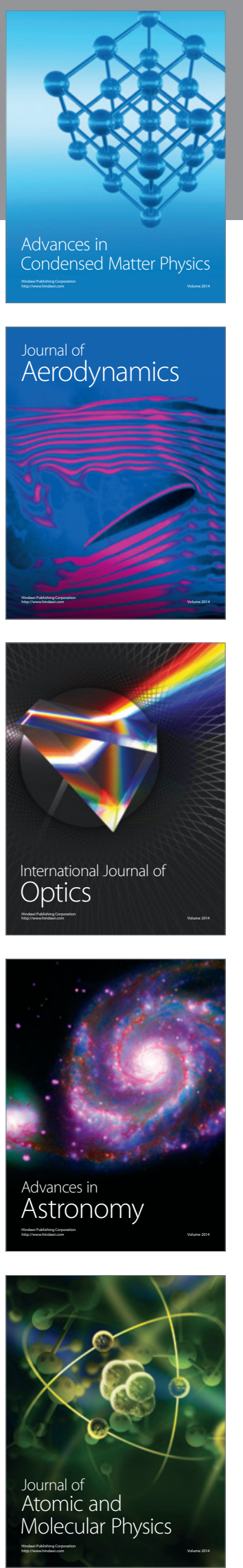